T. Hattori

Nagoya Math. J.

Vol. 145 (1997), 69-98

\title{
DISCRETE SPECTRUM OF MANY BODY SCHRÖDINGER OPERATORS WITH NON-CONSTANT MAGNETIC FIELDS II
}

\author{
TETSUYA HATTORI
}

\section{Introduction}

This paper is continuation from [10], in which we studied the discrete spectrum of atomic Hamiltonians with non-constant magnetic fields and, more precisely, we showed that any atomic system has only finitely many bound states, corresponding to the discrete energy levels, in a suitable magnetic field. In this paper we show another phenomenon in non-constant magnetic fields that any atomic system has infinitely many bound states in a suitable magnetic field.

We consider a following Schrödinger operator of many particle system:

$$
H_{N, Z}(b)=\sum_{j=1}^{N}\left(T_{j}(b)^{2}-\frac{Z}{\left|x^{j}\right|}\right)+\sum_{1 \leq i<j \leq N} \frac{1}{\left|x^{i}-x^{j}\right|}
$$

as a self-adjoint one in $L^{2}\left(\boldsymbol{R}^{3 N}\right)$, where $x=\left(x^{1}, \ldots, x^{N}\right) \in \mathbf{R}^{3 N}, x^{j} \in \mathbf{R}^{3}(1 \leq j$ $\leq N), Z$ is a positive constant, $N \in \mathbf{N}, b \in C^{1}\left(\mathbf{R}^{3}\right)^{3}$, which is real-valued, and

$$
T_{j} \equiv T_{j}(b)=-i \nabla_{j}-b\left(x^{j}\right)(1 \leq j \leq N) .
$$

Here $\nabla_{j}$ denotes the gradient with respect to $x^{j}(1 \leq j \leq N)$. For a vector potential $b \in C^{1}\left(\mathbf{R}^{3}\right)^{3}$, the vector field $\vec{B}(y) \equiv \nabla \times b(y)$ is called the magnetic field. This $H_{N, Z}(b)$ is an energy operator corresponding to an atomic system with Coulomb interactions, in a magnetic field $\nabla \times b$, with an infinitely heavy nucleus of charge $Z$, and with $N$ electrons of charge -1 .

Now our problem is to study the finiteness or the infiniteness of the discrete spectrum of $H_{N, Z}(b)$. This problem has been already studied both in the case without magnetic fields and in the case of constant magnetic fields. As for the case $b=0$, Zhislin showed in [23] that if $Z>N-1$ then the number of the discrete spectrum of $H_{N, Z}(b)$ is infinite, and thereafter in [24] that if $Z<N-1$ then it is

Received May 25, 1995. 
finite. Yafaef asserted in [21] without a proof that it is finite also in the case $Z=$ $N-1$. Thereafter in [22] he proved this is true. We combine these results as follows.

THEOREM 0.1. The number of the discrete spectrum of $H_{N, Z}(0)$ is finite if and only if $Z \leq N-1$.

Next let $b_{c}\left(\in C^{1}\left(\mathbf{R}^{3}\right)^{3}\right)$ give a constant magnetic field, that is, $\nabla \times b_{c}$ is a constant vector field. As for the case $b=b_{c}$, Avron-Herbst-Simon [4] proved that the discrete spectrum of $H_{N, Z}\left(b_{c}\right)$ is infinite if $Z=N-1$. From this result we naturally suggest that it is infinite also in the case $Z \geq N-1$. This is true and easily seen. On the other hand, Vugal'ter-Zhislin [20] asserted (without proof) that the above sufficient condition for the infiniteness is also necessary. This is certainly true and shown by the analogous method as in the non-magnetic case. We combine these results as follows.

THEOREM 0.2. The number of the discrete spectrum of $H_{N, Z}\left(b_{c}\right)$ is finite if and only if $Z<N-1$.

We remark that the above result is independent of strength of magnetic fields and that the difference between the presence and the absence of constant magnetic fields appears only in the case $Z=N-1$, which corresponds to once negatively charged ions.

Now we are interested in the case of non-constant magnetic fields, which has not been studied so deeply (Zhislin [25], [26] treated many body Schrödinger operators with short range scalar potentials and with vector potentials decaying at infinity). In this case the finiteness or the infiniteness of the discrete spectrum of $H_{N, Z}(b)$ depends not only on $N$ and $Z$ but also on magnetic fields. In fact we have the following result in [10].

THEOREM 0.3 For any positive number $\varepsilon$ there exists a vector potential $b_{\varepsilon} \in$ $C^{1}\left(\mathbf{R}^{3}\right)^{3}$, which is independent of $N$ and $Z$, such that the number of the discrete spectrum of $H_{N, Z}(b)$ is finite for $Z \geq \varepsilon$ and $N \geq 1$.

Our main result of this paper is the following theorem, which is contrastive to the above. 
THEOREM 1.1. There exists a vector potential $b_{0} \in C^{1}\left(\mathbf{R}^{3}\right)^{3}$, which gives a perturbed constant magnetic field and is independent of $N$ and $Z$, such that the number of the discrete spectrum of $H_{N, Z}\left(b_{0}\right)$ is infinite for any $N$ and $Z$.

This is another evidence that the finiteness or the infiniteness of the discrete spectrum of $H_{N, Z}(b)$ depends on magnetic field. As stated first, any atomic system has infinitely many bound states in a suitable magnetic fields, even if we add any number of electrons to it.

Also it follows from Theorem 1.1 that the atomic Hamiltonian $H_{N, Z}\left(b_{0}\right)$ has non-empty discrete spectrum, that is related to the problem of the presence or the absence of the descrete spectrum. This problem has been already studied by Ruskai [16], [17], Sigal [18], Lieb [11] and others. Ruskai and Sigal proved that there is no very negative ions, that is, for given $Z$, if $N$ is sufficient large, then $H_{N, Z}(0)$ has no discrete spectrum, and further they studied sufficient conditions for the absence of the discrete spectrum (see also Cycon et al. [7] Chap. 3). Lieb [11] improved their results as follows.

THEOREM 0.4. If $N \geq 2 Z+1$, then $H_{N, Z}(0)$ has no discrete spectrum.

As a consequence of the above theorem, the number of electrons that a nucleus of charge $Z$ can bind is less than $2 Z+1$. Comparing Theorems 0.4 with 1.1 , we see that the existence of the discrete spectrum generically depends on magnetic fields. Moreover, the maximal number of electrons that a nucleus can bind depends not only on the charge of the nucleus but also on the magnetic field.

In $\S 2$ we prepare basic lemmas and Agmon's $K$-function by which we study the bottom of the essential spectrum of $H_{N, Z}(b)$. In $\S 3$ we show $L^{2}$-exponential decay of eigenfunctions, that is due to Agmon [1] and [2]. In $\S 4$ we show an example of the magnetic bottle with infinitely number of discrete spectrum. This vector potential has the property of the main theorem, that is shown in $\S 5$.

\section{Preliminaries}

In this section we make some preparations for the later sections. The aim of this section is to study the location of the infimum of the essential spectrum of $H_{N, Z}(b)$. First we denote the spectrum, the discrete spectrum, the essential spectrum of a self-adjoint operator $H$ by $\sigma(H), \sigma_{d}(H), \sigma_{e}(H)$, respectively, and the cardinal number of a set $Y$ by $\# Y$. Also we put 


$$
\Lambda(H)=\inf \sigma(H) \text { and } \Sigma(H)=\inf \sigma_{e}(H) .
$$

For two vector-valued functions $F$ and $G$, we denote $(F, G)_{L^{2}}=\int_{\mathbf{R}^{3 N}} F \cdot \vec{G} d x$. Now we go on with the argument by restricting $H=H_{N, Z}(b)$. For the sake of convenience we denote $H_{N}=H_{N, Z}(b)$ when $Z$ and $b$ are fixed. Then recalling the notation in $\S 1$, we define the quadratic form

$$
q_{H_{N}}[\phi, \phi]=\sum_{j=1}^{N}\left(T_{j} \phi, T_{j} \phi\right)_{L^{2}}+(V \phi, \psi)_{L^{2}}, q_{H_{N}}[\phi]=q_{H_{N}}[\phi, \phi]
$$

for $\phi, \phi \in C_{0}^{\infty}\left(\mathbf{R}^{3 N}\right)$, where we put

$$
V(x)=\sum_{j=1}^{N}\left(-\frac{Z}{\left|x^{j}\right|}\right)+\sum_{1 \leq i<j \leq N} \frac{1}{\left|x^{i}-x^{j}\right|} .
$$

The infimum of the spectrum and that of the essential spectrum of $H_{N}$ can be expressed as follows (see also Persson [13] and Agmon [1] Theorem 3.2).

LEMma 2.1.

$$
\begin{gathered}
\Lambda\left(H_{N}\right)=\inf \left\{q_{H_{N}}[\phi] ; \phi \in C_{0}^{\infty}\left(\mathbf{R}^{3 N}\right),\|\phi\|_{L^{2}}=1\right\}, \\
\sum\left(H_{N}\right)=\sup _{E: \text { compact }} \inf \left\{q_{H_{N}}[\phi] ; \phi \in C_{0}^{\infty}\left(\mathbf{R}^{3 N} \backslash E\right),\|\phi\|_{L^{2}}=1\right\} .
\end{gathered}
$$

One can show the above equalities in the same way as in the case without magnetic fields (see [1] Theorem 3.2, [2] Theorem 1.6). Next the following localization formula holds as in the case without magnetic fields (see [7] Chap. 3, [8] Lemma 23).

Lemma 2.2 (IMS-localization formula). For a smooth partition of unity $\left\{J_{\alpha}\right\}_{\alpha}$ such that $\Sigma_{\alpha} J_{\alpha}^{2}(x)=1$, the following equality holds:

$$
H_{N}=\sum_{\alpha}\left(J_{\alpha} H_{N} J_{\alpha}-\left|\nabla J_{\alpha}\right|^{2}\right) \text { in the form sense. }
$$

Also we can extend the above two basic lemmas to the case of general scalar potentials (see [10]). Now we define Agmon's function which serves to study the bottom of the essential spectrum of $H_{N}$ (see [1] Chap. 2, [2] §5, [10]).

Definition (Agmon's $K$-function). Let

$$
S^{3 N-1}=\left\{\omega=\left(\omega^{1}, \ldots, \omega^{N}\right) \in \boldsymbol{R}^{3 N} ;|\omega|=1\right\} .
$$

For a subset $U \subset S^{3 N-1}(U \neq \emptyset)$ and for positive numbers $R$ and $\delta$, we put 


$$
\left\{\begin{array}{l}
U_{\delta}=\left\{\omega \in S^{3 N-1} ; \operatorname{dist}(\omega, U)<\delta\right\}, \\
\Gamma\left(U_{\delta}, R\right)=\left\{x \in \mathbf{R}^{3 N} ; x /|x| \in U_{\delta},|x|>R\right\}, \\
K\left(U_{\delta}, R ; H_{N}\right)=\inf \left\{q_{H_{N}}[\phi] ; \phi \in C_{0}^{\infty}\left(\Gamma\left(U_{\delta}, R\right)\right),\|\phi\|_{L^{2}}=1\right\}, \\
K\left(U ; H_{N}\right)=\lim _{\delta \downarrow 0} \lim _{R \uparrow \infty} K\left(U_{\delta}, R ; H_{N}\right) .
\end{array}\right.
$$

One can show the following properties of $K$ in the same way as in the case that $b=0$ ([1] Chap. 2, [2] $\S 5$ and [8]).

LEMMA 2.3. The function $K$ has the following properties.

(i) The value of $K\left(U ; H_{N}\right)$ is the same regardless of the order of the limits.

(ii) The function $K\left(\omega ; H_{N}\right)$ is lower semi-continuous on $S^{3 N-1}$, where $K(\omega$; $\left.H_{N}\right)=K\left(\{\omega\} ; H_{N}\right)$.

(iii) $\sum\left(H_{N}\right)=\min _{\omega \in S^{3 N-1}} K\left(\omega ; H_{N}\right)$.

We remark that Agmon's function is similarly treated also in the case of general scalar potentials (see [10]). For $b \in C^{1}\left(\mathbf{R}^{3}\right)^{3}$, we now define the subsystem of $H_{N}$ as follows.

Definition. For $\omega=\left(\omega^{1}, \ldots, \omega^{N}\right) \in S^{3 N-1}$, let

$$
\begin{gathered}
\left(H_{N}\right)_{\omega}=\sum_{j=1}^{N} T,(b)^{2}+V_{\omega} \text { in } L^{2}\left(\mathbf{R}^{3 N}\right) \text { and } \\
V_{\omega}(x)=\sum_{\omega^{j}=0}\left(-\frac{Z}{\left|x^{j}\right|}\right)+\sum_{\omega^{i}=\omega^{j, 1} \leq i<j \leq N} \frac{1}{\left|x^{i}-x^{j}\right|} .
\end{gathered}
$$

This operator $\left(H_{N}\right)_{\omega}$ is called the subsystem of $H_{N}$ with respect to $\omega \in 3^{3 N-1}$ (see also [10]). Then the next lemma follows from the proof of Lemma 2.5 in [10].

Lemma 2.4. $K\left(\omega ; H_{N}\right)=K\left(\omega ;\left(H_{N}\right)_{\omega}\right)$ for $\omega \in S^{3 N-1}$.

We consider only the case that

$$
b(y)=f(r)\left(-y_{2}, y_{1}, 0\right),
$$

where $y=\left(y_{1}, y_{2}, y_{3}\right) \in \mathbf{R}^{3}, r=|y|, f$ is real-valued function in $C^{1}([0, \infty))$ and $f^{\prime}(0)=0$. We assume that

$$
f(r)-B / 2=C_{0} r^{-\alpha}(\alpha>0, r>>1)
$$

for some constant $C_{0}$ and some positive constant $B$. The constant $C_{0}$ is not necessarily positive. We note this vector potential $b$ gives a perturbed constant magne- 
tic field. The vector potentials presented later in $\S 4$ take the form of (2.4) and satisfy the above assumption. Under the above situation, we study the bottom of the essential spectrum of $T(b)^{2}$ and $H_{N, Z}(b)$. The case of $\alpha>1$ has been already studied in [9] and [10].

THEOREM 2.5. If $\alpha>1$, then

$$
\inf \sigma_{e}\left(T(b)^{2}\right)=B \text { and } \inf \sigma_{e}\left(H_{N, Z}(b)\right)=B+\Lambda\left(H_{N-1, Z}(b)\right) .
$$

Therefore we study only the case that $0<\alpha \leq 1$.

First one can show the following theorem, of which we omit the proof, in the same way as in [9] (Example 2).

THEOREM 2.6. If $0<\alpha \leq 1$, then

$$
\inf \sigma_{e}\left(T(b)^{2}\right)=B \text {. }
$$

Next we consider the bottom of the essential spectrum of atomic Hamiltonians $H_{N, Z}(b)$ in the case $0<\alpha \leq 1$. Then we want to show the following theorem.

TheOREM 2.7. Let $n \geq 2$ and $H_{l}=H_{l, z}(b)(l \in \mathbf{N})$ for short. In the case $0<\alpha$ $\leq 1$, suppose that

$$
\sum\left(H_{l}\right)=\Lambda\left(H_{l-1}\right)+B(1 \leq l \leq n-1),
$$

where we define $H_{l=0}=0$. Then

$$
\sum\left(H_{n}\right) \geq \Lambda\left(H_{l-1}\right)+B .
$$

In order to prove Theorem 2.7 we shall use the following inequality. This appears in [9] and also follows from Theorem 2.9 in [3].

LEMMA 2.8. Let $b(y)=F(y)\left(-y_{2}, y_{1}, 0\right)$, where $y=\left(y_{1}, y_{2}, y_{3}\right) \in \mathbf{R}^{3}$ and $F$ $\in C^{1}\left(\mathbf{R}^{3}\right)$. Also let $\vec{\rho}=\left(y_{1}, y_{2}\right)$ and $\rho=|\vec{\rho}|$. Then

$$
T(b)^{2} \geq 2 F+\rho \frac{\partial}{\partial \rho} F \text { in the form sense. }
$$

In particular, for the vector potential in (2.4), we have

$$
T(b)^{2} \geq 2 f(r)+f^{\prime}(r) \frac{\rho^{2}}{r} \text { in the form sense. }
$$


Proof of Theorem 2.7. By Lemma 2.4,

$$
K\left(\omega ; H_{n}\right)=K\left(\omega ;\left(H_{n}\right)_{\omega}\right) \text { for } \omega \in S^{3 N-1} .
$$

By the property of $K$-function (Lemma 2.3),

$$
\sum\left(H_{n}\right)=\min _{\omega \in S^{3 n-1}} K\left(\omega ; H_{n}\right)=\min _{\omega \in S^{3 n-1}} K\left(\omega ;\left(H_{n}\right)_{\omega}\right) .
$$

Now we will show that

$$
\min _{\omega \in S^{3 n-1}} K\left(\omega ;\left(H_{n}\right)_{\omega}\right) \geq \min _{1 \leq l \leq n}\left(\Lambda\left(H_{n-l}\right)+l B\right) .
$$

By the definition of subsystem, for $\omega=\left(\omega^{1}, \ldots, \omega^{n}\right) \in S^{3 n-1}$, without loss of generality we can assume that

$$
\begin{gathered}
\left(H_{n}\right)_{\omega}=H_{n-l}\left(x^{l+1}, \ldots, x^{n}\right)+\sum_{j=1}^{l} T_{j}^{2}+\sum_{\omega^{i}=\omega^{j} \neq 0,1 \leq i<j \leq l} \frac{1}{\left|x^{i}-x^{j}\right|} \\
\text { and } \omega^{j} \neq 0(j=1, \ldots, l), \omega^{j}=0 \quad(k=l+1, \ldots, n),
\end{gathered}
$$

for some number $l \in\{1, \ldots, n\}$. This implies

$$
\left(H_{n}\right)_{\omega} \geq \Lambda\left(H_{n-l}\right)+\sum_{j=1}^{l} T_{j}^{2} \text { in the form sense. }
$$

We now apply Lemma 2.8 to the vector potential $b$ in (2.4) with the assumption (2.5). If $C_{0} \geq 0\left(C_{0}\right.$ is in (2.5)), then $2 f+f^{\prime}(r) \rho^{2} / r \geq B+C_{0}(2-\alpha) r^{-\alpha} \geq B$ when $r$ is large. If $C_{0}<0$, then $2 f+f^{\prime}(r) \rho^{2} / r \geq 2 f \geq B-\left(-2 C_{0}\right) r^{-\alpha}$ when $r$ is large. Hence, if necessary, by exchanging the constant, we have by Lemma 2.8

$$
\left(T_{j}^{2} \phi, \phi\right)_{L^{2}} \geq B\|\phi\|_{L^{2}}^{2}-\text { (positive constant) }\left(\left|x^{j}\right|^{-\alpha} \phi, \phi\right)_{L^{2}}
$$

for $\phi \in C_{0}^{\infty}\left(\mathbf{R}^{3 n}\right)$. If $x \in \Gamma\left(\omega_{\delta}, R\right)$, then $\left|x^{j}\right|>\left(\left|\omega^{j}\right|-\delta\right)|x|$ for $j \in\{1, \ldots, l\}$. Let $\delta \leq\left(\min _{1 \leq \jmath \leq l}\left|\omega^{j}\right|\right) / 2 \equiv \delta_{1}$. Then $\left|x^{j}\right| \geq \delta_{1}|x| \geq \delta_{1} R$ for $j \in\{1, \ldots, l\}$. Hence, for any $\varepsilon>0$ there exists $R_{\varepsilon}>0$ (which is independent of $\delta$ ) such that

$$
\sum_{j=1}^{n}\left(T_{j}^{2} \phi, \phi\right)_{L^{2}} \geq(l B-\varepsilon)\|\phi\|_{L^{2}}^{2} \text { for } \phi \in C_{0}^{\infty}\left(\Gamma\left(\omega_{\delta}, R\right)\right)
$$

which leads to

$$
\left(\left(H_{n}\right)_{\omega} \phi, \phi\right)_{L^{2}} \geq\left\{\Lambda\left(H_{n-l}\right)+l(B-\varepsilon)\right\}\|\phi\|_{L^{2}}^{2} \text { for } \phi \in C_{0}^{\infty}\left(\Gamma\left(\omega_{\delta}, R\right)\right),
$$

if $R \geq R_{\varepsilon}, \delta \leq \delta_{1}$. Hence by the definition of $K$-function we have

$$
K\left(\omega,\left(H_{n}\right)_{\omega}\right) \geq \Lambda\left(H_{n-l}\right)+l B,
$$


which implies (2.13).

Now suppose (2.8), that is, $\sum\left(H_{l}\right)=\Lambda\left(H_{l-1}\right)+B(1 \leq l \leq n-1)$. Then

$$
\begin{aligned}
n B & =(n-1) B+\sum\left(H_{1}\right) \geq(n-1) B+\Lambda\left(H_{1}\right) \\
& =(n-2) B+\sum\left(H_{2}\right) \geq(n-2) B+\Lambda\left(H_{2}\right) \\
& =\ldots . \\
& =B+\sum\left(H_{n-1}\right) \geq B+\Lambda\left(H_{n-1}\right) .
\end{aligned}
$$

By (2.13) this implies

$$
\min _{1 \leq l \leq n}\left(\Lambda\left(H_{n-l}\right)+l B\right) \geq B+\Lambda\left(H_{n-1}\right) .
$$

Combining (2.12), (2.13) with (2.14) we obtain (2.9).

\section{Decay of eigenfunctions}

In this section we show the $L^{2}$-exponential decay of eigenfunctions in the case of non-constant magnetic fields. We make use of this property in the proof of Theorem 1.1. Since we need only the isotropic $L^{2}$-exponential estimate of eigenfunctions, we do not touch neither the non-isotropic estimate nor the pointwise estimate (for example, see [5], [19]). In the case without magnetic fields. O'Conner [12] and Combes-Thomas [6] showed the (isotropic) $L^{2}$-exponential decay of eigenfunctions for Schrödinger operators under some restriction on the scalar potentials. In [1] and [2] Agmon discussed this property for general Schrödinger operators without magnetic fields. On the other hand, as for the case of constant magnetic fields, Avron-Herbst-Simon [3] (\$8) showed the pointwise estimate of eigenfunctions of atomic Hamiltonians, which leads to the $L^{2}$-estimate of them. One can show the similar result in the general magnetic case as in the case without magnetic fields. We will make it sure in this section by the same method as in [1] (Theorem 1.5 and Chap. 4) or [2] (§4).

First we state our desired theorem as follows.

THEOREM 3.1. Letting $n \geq 1$ and supposing that $\sigma_{d}\left(H_{n}\right) \neq \emptyset$, we pick up a normalized eigenfunction $u(x)$ corresponding to the ground state energy $\Lambda\left(H_{n}\right)$. Then there are some positive constants $\delta_{0}$ and $\delta_{1}$ such that

$$
\int_{\mathbf{R}^{3 n}}|u(x)|^{2} e^{2 \delta_{0}|x|} d x<+\infty \text { and } \sum_{j=1}^{n} \int_{\mathbf{R}^{3 n}}\left|T_{j}(b) u(x)\right|^{2} e^{2 \delta_{1}|x|} d x<+\infty,
$$

where $T_{j}(b)$ is defined in $\S 1$. 
In view of the proof of Theorem 3.1 in this section, we shall see that it is needless to require that $u(x)$ is a ground state. Namely, we can replace $u(x)$ in Theorem 3.1 by the eigenfunctions corresponding to the discrete spectrum below the essential spectrum.

In order to prove Theorem 3.1 we make some preparations. We now consider the following operator:

$$
H=\sum_{j=1}^{n} T_{j}(b)^{2}+V(x) \text { in } L^{2}\left(\mathbf{R}^{3 n}\right),
$$

where $b \in C^{1}\left(\mathbf{R}^{3}\right)^{3}, V \in L_{\text {loc }}^{1}\left(\mathbf{R}^{3 n}\right)$ and $V_{-} \in \mathscr{K}\left(\mathbf{R}^{3 n}\right)$, which are real-valued. Here $V_{-}(x)=\max \{-V(x), 0\}$ and $\mathscr{K}\left(\mathbf{R}^{3 n}\right)$ is Kato class (see [1] Chap. 0, [7] Chap. 1), that is,

$$
\mathscr{K}\left(\mathbf{R}^{3 n}\right)=\left\{u \in L_{\mathrm{loc}}^{1}\left(\mathbf{R}^{3 n}\right) ; \lim _{r \downarrow 0} \sup _{x^{0} \in \mathbf{R}^{3 n}} \int_{\left|x-x^{0}\right| \leq r}|u(x)|\left|x-x^{0}\right|^{2-3 n} d x=0\right\} .
$$

The atomic Hamiltonian $H_{N, Z}(b)$, defined in $\S 1$, is a special case of the above $H$. We remark that the functions in Kato class are $\sum_{j=1}^{n} T_{j}^{2}$-form bounded with the bound zero. Hence for any $\varepsilon>0$ there is a positive constant $C_{\varepsilon}$ such that

$$
\left(V_{-} \phi, \phi\right)_{L^{2}} \leq \varepsilon \sum_{j=1}^{n}\left\|T_{j} \phi\right\|_{L^{2}}^{2}+C_{\varepsilon}\|\phi\|_{L^{2}}^{2}
$$

for $\phi \in C_{0}^{\infty}\left(\mathbf{R}^{3 N}\right)$. For a self-adjoint operator $P$ we denote the domain and the form domain of $P$ by $D(P)$ and $Q(P)$, respectively. We remark that

$$
\begin{gathered}
D(H)=\left\{u \in L^{2}\left(\mathbf{R}^{3 n}\right) ; T_{j} u,|V|^{1 / 2} u, H u \in L^{2}\left(\mathbf{R}^{3 n}\right) \quad(1 \leq j \leq n)\right\}, \\
Q(H)=\left\{u \in L^{2}\left(\mathbf{R}^{3 n}\right) ; T_{j} u,|V|^{1 / 2} u \in L^{2}\left(\mathbf{R}^{3 n}\right) \quad(1 \leq j \leq n)\right\} .
\end{gathered}
$$

The following proposition will be essentially used for the proof of Theorem 3.1.

Proposition 3.2. Let $u \in D(H)$ solve

$$
(H-\mu) u=f \text { in } L^{2}\left(\mathbf{R}^{3 n}\right),
$$

where $\mu \in \mathbf{R}, f \in L^{2}\left(\mathbf{R}^{3 n}\right)$ which is complex-valued. Putting $q=V-\mu$, we assume that there is a positive constant $\lambda$ such that

$$
\sum_{j=1}^{n}\left\|T_{j} \phi\right\|_{L^{2}}^{2}+(q \phi, \phi)_{L^{2}} \geq \lambda\|\phi\|_{L^{2}}^{2} \text { for } \phi \in C_{0}^{\infty}\left(\mathbf{R}^{3 n}\right) .
$$

Further let $h(x)$ be a real-valued Lipschitz function in $\mathbf{R}^{3 n}$ satisfying 


$$
|\nabla h|^{2}<\lambda \quad \text { a.e. }
$$

Then the following inequality holds.

$$
\int_{\mathbf{R}^{3 n}}|u(x)|^{2}\left(\lambda-|\nabla h|^{2}\right) e^{2 h(x)} d x \leq \int_{\mathbf{R}^{3 n}}|f(x)|^{2}\left(\lambda-|\nabla h|^{2}\right)^{-1} e^{2 h(x)} d x
$$

Now we prepare one more lemma as follows (see Agmon [2] Lemma 1.7).

\section{Lemma 3.3. Suppose that}

$$
(H \phi, \phi)_{L^{2}} \geq \int_{\mathbf{R}^{3 n} \backslash K_{0}} c(x)|\phi|^{2} d x \text { for } \phi \in C_{0}^{\infty}\left(\mathbf{R}^{3 n} \backslash K_{0}\right),
$$

where $K_{0}$ is a compact set in $\mathbf{R}^{3 n}$ and $c(x)$ is a real-valued continuous function in $\mathbf{R}^{3 n}$. Then there exists a non-negative function $\chi \in C_{0}^{\infty}\left(\mathbf{R}^{3 n}\right)$ such that

$$
\left(H_{\chi} \phi, \phi\right)_{L^{2}} \geq \int_{\mathbf{R}^{3 n}} c(x)|\phi|^{2} d x \text { for } \phi \in C_{0}^{\infty}\left(\mathbf{R}^{3 n}\right),
$$

where $H_{\chi}=H+\chi$.

For a while admitting Proposition 3.2 and Lemma 3.3, we will show Theorem 3.1 from now on. The following proof is due to Agmon [2] (Theorem 4.3).

Proof of Theorem 3.1. Let $\mu_{1}=\Lambda(H) \in \sigma_{d}(H)$. Also let $u(x)$ be an eigenfunction corresponding to $\Lambda(H)$, namely,

$$
\left(H-\mu_{1}\right) u=0 \text { in } L^{2}\left(\mathbf{R}^{3 n}\right) .
$$

For any $E \in\left(\mu_{1}, \Sigma(H)\right)$ there exists a positive number $R$ such that

$$
(H \phi, \phi)_{L^{2}} \geq E\|\phi\|_{L^{2}}^{2} \text { for } \phi \in C_{0}^{\infty}\left(\Omega_{R}\right),
$$

where $\Omega_{R}=\left\{x \in \mathbf{R}^{3 n} ;|x|>R\right\}$. This follows from Lemma 2.1. By Lemma 3.3, there exists a non-negative function $\chi \in C_{0}^{\infty}\left(\mathbf{R}^{3 n}\right)$ such that

$$
\left(\left(H_{\chi}-\mu_{1}\right) \phi, \phi\right)_{L^{2}} \geq\left(E-\mu_{1}\right)\|\phi\|_{L^{2}}^{2} \text { for } \phi \in C_{0}^{\infty}\left(\mathbf{R}^{3 n}\right),
$$

where $H_{\chi}=H+\chi$. We now pick up a function $\zeta \in C_{0}^{\infty}\left(\mathbf{R}^{3 n}\right)$ satisfying $0 \leq \zeta$ $\leq 1$ and $\zeta=1$ if $|x| \leq R+1 / 2, \zeta=0$ if $|x| \geq R+1$. Using the above function $\zeta$, we put

$$
v(x)=(1-\zeta(x)) u(x) \text { for } x \in \mathbf{R}^{3 n} .
$$


We note that $v \in H_{10 \mathrm{c}}^{1}\left(\mathbf{R}^{3 n}\right)$ and

$$
v(x)=u(x) \text { for }|x| \geq R+1 .
$$

By using the relation that the commutator $\left[T_{j}, \phi\right]=-i\left(\nabla_{j} \phi\right)$ for $\phi \in C^{1}\left(\mathbf{R}^{3 n}\right)$, and by a straightforward manipulation, we have (by (3.7))

$$
\left(H_{\chi}-\mu_{1}\right) v=2 i \sum_{j=1}^{n} \nabla_{j} \zeta \cdot T_{j} u+(\chi(1-\zeta)+\Delta \zeta) u
$$

Let $g=$ [the right-hand side of (3.9)]. Then the function $v$ satisfies

$$
\left(H_{\chi}-\mu_{1}\right) v=g \text { in } L^{2}\left(\mathbf{R}^{3 n}\right) .
$$

Now we apply Proposition 3.2 with

$$
q=V+\chi-\mu_{1}, f=g, \lambda=E-\mu_{1} \text { and } h=(1-\varepsilon) \sqrt{\lambda}|x|(0<\varepsilon<1) .
$$

Note that the assumption of Proposition 3.2 is fulfilled in this case. Then we have

$$
\int_{\mathbf{R}^{3 n}}|v|^{2}\left(\lambda-|\nabla h|^{2}\right) e^{2(1-\varepsilon) \sqrt{\lambda}|x|} d x \leq \int_{\mathbf{R}^{3 n}}|g|^{2}\left(\lambda-|\nabla h|^{2}\right)^{-1} e^{2(1-\varepsilon) \sqrt{\lambda}|x|} d x .
$$

Since $g$ is compactly supported and $\lambda-|\nabla h|^{2} \geq \varepsilon \lambda$, we have

$$
\int_{\mathbf{R}^{3 n}}|v(x)|^{2} e^{2(1-\varepsilon) \sqrt{\lambda}|x|} d x<+\infty,
$$

which implies by (3.8)

$$
\int_{\Omega_{R+1}}|u(x)|^{2} e^{2(1-\varepsilon) \sqrt{\lambda}|x|} d x<+\infty .
$$

Hence letting $\delta_{0}=(1-\varepsilon) \sqrt{\lambda}$ we have

$$
\int_{\mathbf{R}^{3 n}}|u(x)|^{2} e^{2 \delta_{0}|x|} d x<+\infty
$$

Next we show the second part of (3.1). We pick up a function $\chi_{1} \in$ $C_{0}^{\infty}\left(\mathbf{R}^{3 n}\right)$ such that $0 \leq \chi_{1} \leq 1$, and $\chi_{1}(x)=1 \quad(|x| \leq 1), \chi_{1}(x)=0 \quad(|x| \geq 2)$. Letting $\chi_{m}(x)=\chi_{1}(x / m)$ and $\alpha=1-2 \varepsilon(\varepsilon$ is in (3.11)), we define functions $v_{m} \in L^{2}\left(\mathbf{R}^{3 n}\right)$ by

$$
v_{m}(x)=\chi_{m}(x)^{2} u(x) e^{\alpha \sqrt{\lambda}|x|}(m \in \mathbf{N}) .
$$

We remark that $v_{m} \in Q(H)$ (the form domain of $H$ ). Since $\left(H-\mu_{1}\right) u=0$ in $L^{2}\left(\mathbf{R}^{3 n}\right)$ 


$$
\sum_{j=1}^{n}\left(T_{j} u, T_{j} v_{m}\right)_{L^{2}}+\left(V u, v_{m}\right)_{L^{2}}=\mu_{1}\left(u, v_{m}\right)_{L^{2}}(m \in \mathbf{N}) .
$$

By a simple computation,

$$
\begin{aligned}
\left(T, u, T_{j} v_{m}\right)_{L^{2}}= & \int_{\mathbf{R}^{3 n}} \chi_{m}^{2} e^{\alpha \sqrt{\lambda}|x|}\left|T_{j} u\right|^{2} d x+i \alpha \sqrt{\lambda}\left(T_{j} u, \chi_{m}^{2} u \frac{x}{\left.|x|^{\alpha \sqrt{\lambda}|x|}\right)_{L^{2}}}\right. \\
& +\frac{2 i}{m}\left(T_{j} u, u e^{\alpha \sqrt{\lambda}|x|} \chi_{m}\left(\nabla_{j} \chi_{1}\right)(x / m)\right)_{L^{2}}
\end{aligned}
$$

Hence we have by (3.14)

$$
-i \alpha \sqrt{\lambda} \sum_{j=1}^{n}\left(T_{j} u, \chi_{m}^{2} u \frac{x}{|x|} e^{\alpha \sqrt{\lambda}|x|}\right)_{L^{2}}-\frac{2 i}{m} \sum_{j=1}^{n}\left(T_{j} u, u e^{\alpha \sqrt{\lambda}|x|} \chi_{m}\left(\nabla_{j} \chi_{1}\right)(x / m)\right)_{L^{2}} .
$$

By using (3.12), it is easy to see that the first, third, fourth term of the right-hand side of (3.15) are uniformly bounded in $m$. As for the second term of (3.14), by the assumption $V_{-} \in \mathscr{K}\left(\mathbf{R}^{3 n}\right)$ and (3.2),

$$
\begin{aligned}
-\left(V u, v_{m}\right)_{L^{2}} \leq{\overline{\left(V_{-} v_{m}, u\right)_{L^{2}}}} & \leq \frac{1}{4} \sum_{j=1}^{n}\left\|T_{j}\left(u e^{\alpha \sqrt{\lambda}|x| / 2} \chi_{m}\right)\right\|_{L^{2}}^{2}+O(1) \\
& \leq \frac{1}{2} \sum_{j=1}^{n} \int_{\mathbf{R}^{3 n}} \chi_{m}^{2} e^{\alpha \sqrt{\lambda}|x|}\left|T_{j} u\right|^{2} d x+O(1) .
\end{aligned}
$$

Here we have used the first part of (3.1) which we have already shown. Combining (3.15) with (3.16) yields

$$
\int_{\mathbf{R}^{3 n}} \chi_{m}^{2} e^{\alpha \sqrt{\lambda}|x|}\left|T_{j} u\right|^{2} d x=O(1) \quad(1 \leq j \leq n)
$$

which is uniformly in $m$. Letting $m \rightarrow \infty$ we have

$$
\int_{\mathbf{R}^{3 n}} e^{\alpha \sqrt{\lambda}|x|}|T, u|^{2} d x=O(1) \quad(1 \leq j \leq n)
$$

We have only to put $\delta_{1}=\alpha \sqrt{\lambda} / 2$.

To complete the proof of Theorem 3.1 we prove Proposition 3.2 and Lemma 3.3. These proofs are due to Agmon [1] (Theorem 1.5) and [2] (Lemma 1.7).

Proof of Lemma 3.3. Pick up non-negative functions $\xi_{0} \in C_{0}^{\infty}\left(\mathbf{R}^{3 n}\right)$ and $\xi_{1} \in$ 
$C^{\infty}\left(\mathbf{R}^{3 n}\right)$ such that $\xi_{1}=0$ in a neighbourhood of $K_{0}$ and $\xi_{0}^{2}+\xi_{1}^{2}=1$ on $\mathbf{R}^{3 n}$. By IMS-localization formula (Lemma 2.2) and by the hypothesis we have, for $\phi \in$ $C_{0}^{\infty}\left(\mathbf{R}^{3 n}\right)$,

$$
\begin{aligned}
(H \phi, \phi)_{L^{2}} & =\sum_{j=0}^{1}\left(H \xi_{j} \phi, \xi_{j} \phi\right)_{L^{2}}-\left(\left(\left|\nabla \xi_{0}\right|^{2}+\left|\nabla \xi_{1}\right|^{2}\right) \phi, \phi\right)_{L^{2}} \\
& \geq\left(c(x) \xi_{1} \phi, \xi_{1} \phi\right)_{L^{2}}+\Lambda(H)\left\|\xi_{0} \phi\right\|_{L^{2}}^{2}-\left(\left(\left|\nabla \xi_{0}\right|^{2}+\left|\nabla \xi_{1}\right|^{2}\right) \phi, \phi\right)_{L^{2}} \\
& =\left(c_{1}(x) \phi, \phi\right)_{L^{2}}
\end{aligned}
$$

where we set

$$
c_{1}(x)=\xi_{1}(x)^{2} c(x)+\Lambda(H) \xi_{0}(x)^{2}-\left(\left|\nabla \xi_{0}\right|^{2}+\left|\nabla \xi_{1}\right|^{2}\right) .
$$

Since $c_{1}(x)=c(x)$ when $|x|$ is sufficiently large, it is easy to see that there is a non-negative function $\chi \in C_{0}^{\infty}\left(\mathbf{R}^{3 n}\right)$ such that $c_{1}(x)+\chi(x) \geq c(x)$ on $\mathbf{R}^{3 n}$. This completes the proof.

Proof of Proposition 3.2. First, by the assumption,

$$
\sum_{j=1}^{n}\left(T_{j} u, T_{j} \phi\right)_{L^{2}}+(q u, \phi)_{L^{2}}=(f, \phi)_{L^{2}}
$$

for $\phi \in C_{0}^{\infty}\left(\mathbf{R}^{3 n}\right)$. Let

$$
Y=L_{0}^{\infty}\left(\mathbf{R}^{3 n}\right) \cap\left\{u ; T_{j} u \in L^{2}\left(\mathbf{R}^{3 n}\right), 1 \leq j \leq n\right\} \subset L^{2}\left(\mathbf{R}^{3 n}\right) .
$$

By using a mollifier and by taking the limit, (3.17) holds also for $\phi \in Y$.

Let $\phi$ be real-valued Lipschitz function with compact support and $u_{\varepsilon}=u /$ ( 1 $\left.+\varepsilon|u|^{2}\right)\left(\varepsilon\right.$ is an arbitrary positive number). As is easily seen, $u_{\varepsilon} \psi^{2} \in Y$. We note that

$$
u_{\varepsilon} \rightarrow u \text { in } H_{\mathrm{loc}}^{1} \text {, and } T_{j} u_{\varepsilon} \rightarrow T_{j} u \text { in } L_{\mathrm{loc}}^{2} \text { as } \varepsilon \downarrow 0 .
$$

By substituting $\phi=u_{\varepsilon} \phi^{2}$ into (3.17), we have

$$
\sum_{j=1}^{n}\left(T_{j} u, T_{j}\left(u_{\varepsilon} \psi^{2}\right)\right)_{L^{2}}+\left(q u, u_{\varepsilon} \psi^{2}\right)_{L^{2}}=\left(f, u_{\varepsilon} \phi^{2}\right)_{L^{2}}
$$

Rewrite $u=u_{\varepsilon}+\left(u-u_{\varepsilon}\right)$ and take the real part of the both sides in (3.19). Then

$$
\operatorname{Re} \sum_{j=1}^{n}\left(T, u_{\varepsilon}, T_{j} u_{\varepsilon} \psi^{2}\right)_{L^{2}}+\left(q u_{\varepsilon}, u_{\varepsilon} \phi^{2}\right)_{L^{2}}=I_{\varepsilon}+\operatorname{Re}\left(f, u_{\varepsilon} \phi^{2}\right)_{L^{2}}
$$

where 


$$
I_{\varepsilon}=-\operatorname{Re} \sum_{j=1}^{n}\left(T_{j}\left(u-u_{\varepsilon}\right), T_{j} u_{\varepsilon} \phi^{2}\right)_{L^{2}}-\left(q\left(u-u_{\varepsilon}\right), u_{\varepsilon} \phi^{2}\right)_{L^{2}}
$$

Since $-q\left(u-u_{\varepsilon}\right) \bar{u}_{\varepsilon} \leq q_{-}\left(u-u_{\varepsilon}\right) \bar{u}_{\varepsilon}\left(q_{-}(x)=\max \{-q(x), 0\}\right)$,

$$
\begin{aligned}
I_{\varepsilon} \leq & \sum_{j=1}^{n}\left\|T_{j}\left(u-u_{\varepsilon}\right)\right\|_{L^{2}(\Omega)} \cdot\left\|T_{j}\left(u_{\varepsilon} \phi^{2}\right)\right\|_{L^{2}(\Omega)} \\
& +\left(q_{-}\left(u-u_{\varepsilon}\right) \psi,\left(u-u_{\varepsilon}\right) \phi\right)_{L^{2}\left(\mathbf{R}^{3 n)}\right.}^{1 / 2} \cdot\left(q_{-} u_{\varepsilon} \psi, u_{\varepsilon} \psi\right)_{L^{2}\left(\mathbf{R}^{3 n}\right)}^{1 / 2},
\end{aligned}
$$

where $\Omega=\operatorname{supp} \phi$ and we have used Schwarz inequality. As is easily seen by (3.18), the first term of the right-hand side of (3.20) converges to zero as $\varepsilon$ tends down to zero. We notice that $q_{-} \in \mathscr{K}\left(\mathbf{R}^{3 n}\right)$ is $\sum_{j} T_{j}^{2}$ relatively form bounded with the bound zero, and that

$$
\begin{aligned}
\left\|T_{j} \phi \phi\right\|_{L^{2}}^{2} & \leq 2\left(\left\|\phi T_{j} \phi\right\|_{L^{2}}^{2}+\left\|\left(\nabla_{j} \phi\right) \phi\right\|_{L^{2}}^{2}\right) \\
& \leq \beta_{0}\left\|T_{j} \phi\right\|_{L^{2}(\Omega)}^{2}+\beta_{1}\|\phi\|_{L^{2}(\Omega)}^{2}
\end{aligned}
$$

for $\phi=u_{\varepsilon}$ or $\phi=u-u_{\varepsilon}$. Here $\beta_{0}$ and $\beta_{1}$ are some positive constants. Hence

$$
\begin{aligned}
\left(q_{-} u_{\varepsilon} \psi, u_{\varepsilon} \psi\right)_{L^{2}} & \leq \sum_{j=1}^{n}\left\|T_{j} u_{\varepsilon} \psi\right\|_{L^{2}}^{2}+C_{1}\left\|u_{\varepsilon} \psi\right\|_{L^{2}}^{2} \\
& \leq \beta_{0} \sum_{j=1}^{n}\left\|T_{j} u_{\varepsilon}\right\|_{L^{2}(\Omega)}^{2}+\left(n \beta_{1}+\sup _{x \in \Omega}|\phi(x)|\right)\left\|u_{\varepsilon}\right\|_{L^{2}(\Omega)}^{2} \\
& <+\infty
\end{aligned}
$$

for some positive constant $C_{1}$, which is independent of $\varepsilon<1$. Hence by using the same method as above we have

$$
\text { [the second term of }(3.21)] \leq \beta_{3}\left[\sum_{j=1}^{n}\left\{\left\|T_{j}\left(u-u_{\varepsilon}\right)\right\|_{L^{2}(\Omega)}^{2}+\left\|u-u_{\varepsilon}\right\|_{L^{2}(\Omega)}^{2}\right\}\right]^{1 / 2}
$$

for some positive constant $\beta_{3}$. By (3.18) again it follows that the second term of (3.21) converges to zero as $\varepsilon$ tends down to zero. Hence we have

$$
\lim _{\varepsilon \downarrow 0} \sup _{\varepsilon} \leq 0
$$

In view of the identity

$$
\operatorname{Re} \int_{\mathbf{R}^{3 n}} T_{j} v \cdot \overline{T_{j}\left(\phi^{2} v\right)} d x=\int_{\mathbf{R}^{3 n}}\left\{\left|T_{j}(\phi v)\right|^{2}-|v|^{2}\left|\nabla_{j} \phi\right|^{2}\right\} d x \text { for } v \in D(H),
$$

by using (3.22) and (3.20) we have

$$
\lim _{\varepsilon \downarrow 0} \sup _{j=1}\left\{\sum_{j=1}^{n}\left\|T_{j} \psi u_{\varepsilon}\right\|_{L^{2}}^{2}+\left(q u_{\varepsilon} \psi, u_{\varepsilon} \psi\right)_{L^{2}}-\left\|u_{\varepsilon} \nabla \psi\right\|_{L^{2}}^{2}-\operatorname{Re}\left(f, u_{\varepsilon} \psi^{2}\right)_{L^{2}}\right\} \leq 0
$$


By (3.18) and the assumption (3.4),

$$
\lambda\|\phi u\|_{L^{2}}^{2}-\|u \nabla \phi\|_{L^{2}}^{2} \leq \operatorname{Re}\left(f, u \phi^{2}\right)_{L^{2}}
$$

Now let $g$ and $\chi$ be real-valued Lipschitz functions such that $\chi$ is compactly supported, $0 \leq \chi \leq 1$ on $\mathbf{R}^{3 n}$ and $|\nabla g|<\lambda$ a.e. Then we can put $\phi=e^{\mathrm{g}} \chi$ in (3.23). Using Schwarz inequality for

$$
\left(f, u e^{2 g} \chi^{2}\right)_{L^{2}}=\left(u\left(\lambda-|\nabla g|^{2}\right)^{1 / 2} e^{g} \chi, f\left(\lambda-|\nabla g|^{2}\right)^{-1 / 2} e^{g} \chi\right)_{L^{2}},
$$

we have by (3.23)

$$
\begin{aligned}
\int_{\mathbf{R}^{3 n}}|\chi u|^{2} e^{2 g}\left(\lambda-|\nabla g|^{2}\right) d x \leq & \left\{\int|\chi u|^{2} e^{2 g}\left(\lambda-|\nabla g|^{2}\right) d x\right\}^{1 / 2}\left\{\int|f \chi|^{2} e^{2 g}\left(\lambda-|\nabla g|^{2}\right)^{-1} d x\right\}^{1 / 2} \\
& +\int|u|^{2} e^{2 g}\left(|\nabla \chi|^{2}+2 \chi|\nabla g \cdot \nabla \chi|\right) d x .
\end{aligned}
$$

In view of the fact that

$$
a \leq \sqrt{a} \sqrt{b}+c(a, b, c>0) \text { implies } a \leq b+2 c,
$$

we have

$$
\begin{aligned}
\int_{\mathbf{R}^{3 n}}|\chi u|^{2} e^{2 g}\left(\lambda-|\nabla g|^{2}\right) d x \leq & \int_{\mathbf{R}^{3 n}}|f \chi|^{2} e^{2 g}\left(\lambda-|\nabla g|^{2}\right)^{-1} d x \\
& +2 \int_{\mathbf{R}^{3 n}}|u|^{2} \chi^{2} e^{2 g}\left(|\nabla \chi|^{2}+2 \chi|\nabla \chi \cdot \nabla g|\right) d x
\end{aligned}
$$

From now on we will show (3.6) by using (3.24) and by taking the limit. Letting $\Omega_{j}=\left\{x \in \mathbf{R}^{3 n} ;|x| \leq j\right\}$, we define functions $\eta(t)$ and $\chi_{j}(x)$ by

$$
\eta(t)=\left\{\begin{array}{l}
t \text { if } t \in[0,1], \\
1 \text { if } t \in(1, \infty),
\end{array} \text { and } \chi_{j}(x)=\eta\left(\sqrt{\lambda} \operatorname{dist}\left(x, \Omega_{j}^{c}\right)\right) .\right.
$$

Then $\chi_{j}$ is a Lipschitz function and

$$
\left|\chi_{j}(x)-\chi_{j}(y)\right| \leq \sqrt{\lambda}|x-y| \text { and }\left|\nabla \chi_{j}(x)\right| \leq \sqrt{\lambda} \text { a.e. }
$$

We define the function $h_{k}(x)$ by $h_{k}(x)=\min \{h(x), k\}(k \in \mathbf{N})$. Then $h_{k}$ is a Lipschitz function and (by (3.5)) $\left|\nabla h_{k}\right|^{2}<\lambda$ a.e. Hence we can put $g=h_{k}$ and $\chi=\chi_{j}$ in $(3.24)$ :

$$
\begin{aligned}
\int_{\mathbf{R}^{3 n}}\left|\chi_{j} u\right|^{2} e^{2 h_{k}}\left(\lambda-\left|\nabla h_{k}\right|^{2}\right) d x & \leq \int_{\mathbf{R}^{3 n}}\left|f \chi_{j}\right|^{2} e^{2 h_{k}}\left(\lambda-\left|\nabla h_{k}\right|^{2}\right) d x \\
& +\int_{\mathbf{R}^{3 n}}|u|^{2} \chi_{j}^{2} e^{2 h_{k}}\left(\left|\nabla \chi_{j}\right|^{2}+2 \chi_{j}\left|\nabla h_{k} \cdot \nabla \chi_{j}\right|\right) d x
\end{aligned}
$$


Since $\chi_{j} \rightarrow 1, \nabla \chi_{j} \rightarrow 0$ and $h_{k} \rightarrow h, \nabla h_{k} \rightarrow \nabla h$ in pointwise sense, letting first $j \rightarrow \infty$ and next $k \rightarrow \infty$ in (3.25), we obtain

$$
\int_{\mathbf{R}^{3 n}}|u|^{2} e^{2 h}\left(\lambda-|\nabla h|^{2}\right) d x \leq \int_{\mathbf{R}^{3 n}}|f|^{2} e^{2 h}\left(\lambda-|\nabla h|^{2}\right) d x
$$

\section{Magnetic bottle}

In this section we show an example of the magnetic bottle, which will be used for the proof of Theorem 1.1. Here a vector potential $b \in C^{1}\left(\mathbf{R}^{3}\right)^{3}$ is called a magnetic bottle of the first (respectively second) [respectively third] kind if and only if $T(b)^{2}$ has some non-zero eigenvalue (respectively $T(b)^{2}$ has pure point spectrum) [respectively $T(b)^{2}$ has compact resolvent] (see [3] 11 and $\S 2$ ), where $T(b)=-i \nabla_{y}-b(y) \quad\left(y \in \mathbf{R}^{3}\right)$. Throughout this section we denote $y=\left(y_{1}, y_{2}, z\right)$ $\in \mathbf{R}^{3}, \vec{\rho}=\left(y_{1}, y_{2}\right), r=|y|, \rho=|\vec{\rho}|$.

Now we want to show the following theorem.

THEOREM 4.1. There exists a vector potential $b_{0} \in C^{1}\left(\mathbf{R}^{3}\right)^{3}$ such that

$$
\# \sigma_{d}\left(T\left(b_{0}\right)^{2}\right)=+\infty,
$$

that is, $b_{0}$ is a magnetic bottle of the first and second kind.

This magnetic bottle $b_{0}$ will be constructed as a perturbation of $b_{c}(y)$, which appears in $\S 1$ and gives a constant magnetic field. Therefore this $b_{0}$ is not a magnetic bottle of the third kind. We notice that the previous step of this theorem appears in [9] Example 4. By min-max principle and Rayleigh-Ritz method (see [14] X III), we have only to prove the following proposition, which plays an important role in the proof of Theorem 1.1 .

Proposition 4.2. There exist a positive constant $\varepsilon_{0}$, a vector potential $b_{0} \in$ $C^{1}\left(\mathbf{R}^{3}\right)^{3}$ and a sequence of functions $\left\{\phi_{m}\right\}_{m} \subset C^{1}\left(\mathbf{R}^{3}\right) \cap Q\left(T^{2}\right)$ such that

$$
\left(\phi_{i}, \phi_{j}\right)_{L^{2}\left(\mathbf{R}^{3}\right)}=\delta_{i j} \text { and }\left\|T \phi_{m}\right\|_{L^{2}\left(\mathbf{R}^{3}\right)}^{2} \leq B-\varepsilon_{0} m^{-1 / 4}(m>>1),
$$

where $Q\left(T^{2}\right)$ denotes the form domain of $T^{2}, B=\inf \sigma_{e}\left(T^{2}\right)$ and $\varepsilon_{0}$ is independent of $m$. Moreover, there is a subsequence of $\left\{\phi_{m}\right\}_{m}$, denoted by $\left\{\phi_{m_{j}}\right\}$, satisfying

$$
\left(T \psi_{m_{i}}, T \psi_{m_{j}}\right)_{L^{2}\left(\mathbf{R}^{3}\right)}=0 \text { if } i \neq j .
$$

We start the proof of Proposition 4.2. Pick up a real-valued function $\eta_{0} \in$ 
$C_{0}^{\infty}(1 \leq z \leq 2)$, which is normalized as $\left\|\eta_{0}\right\|_{L^{2}(\mathbf{R})}=1$. Letting

$$
c_{0}=\left\|\eta_{0}^{\prime}\right\|_{L^{2}(\mathbf{R})}^{2} \text { and } B>2+\frac{64}{9} c_{0}
$$

we define the function $f(\zeta)$ by

$$
f(\zeta)=\left\{\begin{array}{l}
\frac{B}{2}-\zeta^{-1 / 2}(\zeta \geq 2), \\
\frac{B}{2}-\frac{1}{\sqrt{2}}-\frac{\zeta(2-\zeta)}{8 \sqrt{2}}(1<\zeta<2), \\
\frac{B}{2}-\frac{9}{8 \sqrt{2}}(0 \leq \zeta \leq 1) .
\end{array}\right.
$$

Then $f \in C^{1}([0, \infty)), f^{\prime}(\zeta) \geq 0$ and $f$ is real-valued. By using this function $f(\zeta)$, we define the vector potential $b_{0}(y)$ by

$$
b_{0}(y)=f(r)\left(-y_{2}, y_{1}, 0\right) \quad\left(y \in \mathbf{R}^{3}\right) .
$$

We note that $b_{0}(y)$ gives a perturbed constant magnetic field. We will show this vector potential $b_{0}$ is desired one.

Remark 4.3. We want to make $c_{0}$ as small as possible. By the min-max principle for the operator $-d^{2} / d t^{2}$ in $L^{2}(0,1)$ with the Dirichlet boundary condition, we can take $c_{0} \sim \pi^{2}$. Hence we can take $B \geq 2+64 \pi^{2} / 9 \sim 72.2$.

LEMMA 4.4. Let $G_{f}(y)=2 f(r)+f^{\prime}(r) \rho^{2} / r$. Then the following inequality holds:

$$
G_{f}(y) \leq\left\{\begin{array}{l}
B-\frac{3 r^{-1 / 2}}{2}(r \geq 2), \\
B+\frac{\left(2 r^{2}-3 r-8\right)}{4 \sqrt{2}}(1<r<2), \\
B-\frac{9}{4 \sqrt{2}}(0 \leq r \leq 1) .
\end{array}\right.
$$

Definition. Using the cylindrical coordinate $((\rho, \theta), z)$, we define

$$
\begin{aligned}
\phi_{m}(\vec{\rho}, z) & =\beta_{m}(z) e^{i m \theta} \rho^{m} \exp \{-F(\rho, z)\} \\
& =\beta_{m}(z)\left(y_{1}+i y_{2}\right)^{m} \exp \{-F(\rho, z)\} \quad(m \in \mathbf{N}),
\end{aligned}
$$

where 


$$
F(\rho, z)=\int_{0}^{\rho} f\left(\sqrt{t^{2}+z^{2}}\right) t d t
$$

and $\beta_{m}(z)$ is the normalizing constant in $L^{2}\left(\mathbf{R}^{2}\right)$, namely,

$$
\beta_{m}(z)^{2}=\left[2 \pi \int_{0}^{\infty} \rho^{2 m+1} \exp \{-2 F(\rho, z)\} d \rho\right]^{-1}(m \in \mathbf{N}) .
$$

Also letting

$$
\eta_{s}(z)=s^{-1 / 2} \eta_{0}(z / s)(s>1)
$$

for the above $\eta_{0} \in C_{0}^{\infty}(1 \leq z \leq 2)$, we define the function $\phi_{m}^{s}(y)$ by

$$
\phi_{m}^{s}(y)=\eta_{s}(z) \phi_{m}(\vec{\rho}, z) \quad(s>1, m \in \mathbf{N}) .
$$

We shall get the desired sequence of functions in the above form (4.9).

LEMMA 4.5. These functions $\phi_{m}^{s}$ and $\phi_{m}$ have the following properties.

(i) $\phi_{m}^{s} \in C^{1}\left(\mathbf{R}^{3}\right) \cap Q\left(T\left(b_{0}\right)^{2}\right)$, where $Q\left(T\left(b_{0}\right)^{2}\right)$ denotes the form domain of $T\left(b_{0}\right)^{2}$.

(ii) $\left[T\left(b_{0}\right)^{2}-\left(-\partial^{2} / \partial z^{2}\right)\right] \phi_{m}=G_{f}(y) \phi_{m}$, and so $\left[T\left(b_{0}\right)^{2}-\left(-\partial^{2} / \partial z^{2}\right)\right] \phi_{m}^{s}$ $=G_{f}(y) \phi_{m}^{s}$, where $G_{f}(y)$ is defined in Lemma 4.4 .

(iii) $\left(\phi_{m}, \phi_{m^{\prime}}\right)_{L^{2}\left(\mathbf{R}^{2}\right)}=\delta_{m m^{\prime}}$, so $\left\|\phi_{m}^{s}\right\|_{L^{2}\left(\mathbf{R}^{3}\right)}=1,\left(\phi_{m}^{s}, \phi_{m^{\prime}}^{s^{\prime}}\right)_{L^{2}\left(\mathbf{R}^{3}\right)}=0$ if $m \neq m^{\prime}$.

Proof of Lemma 4.5. (iii) is easily obtained. In view of the form domain of $T\left(b_{0}\right)^{2}$ :

$$
Q\left(T\left(b_{0}\right)^{2}\right)=\left\{u \in L^{2}\left(\mathbf{R}^{3}\right) ; T\left(b_{0}\right) u \in L^{2}\left(\mathbf{R}^{3}\right)\right\},
$$

one can easily obtain (i). By using the equality

$$
\begin{aligned}
T\left(b_{0}\right)^{2}-\left(-\partial^{2} / \partial z^{2}\right) & =-\partial^{2} / \partial y_{1}^{2}-\partial^{2} / \partial y_{2}^{2}+2 i b_{0} \cdot \nabla+i\left(\operatorname{div} b_{0}\right)+\left|b_{0}\right|^{2} \\
& =-\partial^{2} / \partial y_{1}^{2}-\partial^{2} / \partial y_{2}^{2}+2 i b_{0} \cdot \nabla+\left|b_{0}\right|^{2}
\end{aligned}
$$

and

$$
\partial_{\rho} \phi_{m}=(m / \rho-f(r) \rho) \phi_{m}, \partial_{\theta} \phi_{m}=i m \phi_{m}(m \in \mathbf{N}),
$$

it is easy to show (ii) by a straightforward calculation.

Lemma 4.6. For any $R>0$ there exists a positive constant $C_{B}(R)$ such that

$$
\beta_{m}(z)^{2} \leq C_{B}(R) R^{-2 m-1} \text { (uniformly in } z ; m \in \mathbf{N} \text { ). }
$$


Proof. Since $f(\zeta) \leq B / 2$, we have by (4.7)

$$
\beta_{m}(z)^{-2}=2 \pi \int_{0}^{\infty} \rho^{2 m+1} e^{-2 F(\rho, z)} d \rho \geq 2 \pi \int_{R}^{\infty} \rho^{2 m+1} e^{-B \rho^{2} / 2} d \rho \geq 2 \pi R^{2 m+1} \int_{R}^{\infty} e^{-B \rho^{2} / 2} d \rho .
$$

Putting $C_{B}(R)=\left(2 \pi \int_{R}^{\infty} e^{-B \rho^{2} / 2} d \rho\right)^{-1}$, we have the desired inequality.

Let $k=\left\{\frac{2}{c_{0}} \sqrt{\frac{2}{B-2}}\right\}^{1 / 4}, s=s(m, k)=k^{-1} m^{1 / 8}$ and $\phi_{m}=\phi_{m}^{s(m, k)}$. Then we can estimate $\left\|\frac{\partial \phi_{m}}{\partial z}\right\|_{L^{2}\left(\mathbf{R}^{3}\right)}^{2}$ as follows. This estimation serves to estimate the quadratic form of $T\left(b_{0}\right)^{2}$.

Lemma 4.7. For any $\varepsilon>0$, the following inequality holds:

$$
\begin{aligned}
\left\|\frac{\partial \phi_{m}}{\partial z}\right\|_{L^{2}\left(\mathbf{R}^{3}\right)}^{2} & \leq(1+\varepsilon)\left\{c_{0} k^{2}+\frac{2}{k^{2}} \sqrt{\frac{2}{B-2}}\right\}(m+1)^{-1 / 4}+c(B, k) m^{-1} \\
& =2(1+\varepsilon) \sqrt{2 c_{0}}\left(\frac{2}{B-2}\right)^{1 / 4}(m+1)^{-1 / 4}+c(B, k) m^{-1}
\end{aligned}
$$

when $m$ is sufficiently large, where $c(B, k)$ is some positive constant depending on $B$ and $k$, and $c_{0}$ appears in (4.3).

To prove Lemma 4.7 we prepare several lemmas as follows.

Lemma 4.8. The normalizing constant $\beta_{m}(z)$ satisfies the following equality.

$$
\beta_{m}^{\prime}(z)=2 \pi \beta_{m}(z)^{3} z \int_{0}^{\infty}(f(r)-f(|z|)) \rho^{2 m+1} e^{-2 F(\rho, z)} d \rho .
$$

Lemma 4.9. Let $g(\zeta)=f(\zeta)-B / 2$. Then the following equality holds.

$$
\begin{aligned}
\int_{\mathbf{R}^{2}}\left|\frac{\partial \phi_{m}}{\partial z}\right|^{2} d \vec{\rho}= & |z|^{2}\left[2 \pi \beta_{m}(z)^{2} \int_{0}^{\infty} g(r)^{2} \rho^{2 m+1} e^{-2 F(\rho, z)} d \rho\right. \\
& \left.-\left\{2 \pi \beta_{m}(z)^{2} \int_{0}^{\infty} g(r) \rho^{2 m+1} e^{-2 F(\rho, z)} d \rho\right\}^{2}\right] .
\end{aligned}
$$

LEMMA 4.10. The first term of the right-hand side of (4.12) is estimated as follows. 


$$
2 \pi \beta_{m}(z)^{2} \int_{0}^{\infty} g(r)^{2} \rho^{2 m+1} e^{-2 F(\rho, z)} d \rho \leq \sqrt{B / 2} m^{-1 / 2}
$$

Lemma 4.11. Let $s=k^{-1} m^{-1 / 8}$. Then the second term of the right-hand side of (4.12) is estimated as follows.

$$
\begin{aligned}
& 2 \pi \beta_{m}(z)^{2} \int_{0}^{\infty}(-g(r)) \rho^{2 m+1} e^{-2 F(\rho, z)} d \rho \\
\geq & \left(\frac{B-2}{2}\right)^{1 / 4}(m+1)^{-1 / 4}-c_{1}(B, k) m^{-1}
\end{aligned}
$$

for $m>1, s \leq z \leq 2 s$, where $c_{1}(B, k)$ is some positive constant depending on $B$ and $k$.

Admitting Lemmas 4.8-11 for the moment, we prove Lemma 4.7.

Proof of Lemma 4.7. By a simple calculation,

$$
\left\|\frac{\partial \phi_{m}^{s}}{\partial z}\right\|_{L^{2}\left(\mathbf{R}^{3}\right)}^{2}=\left\|\eta_{s}^{\prime} \phi_{m}\right\|_{L^{2}\left(\mathbf{R}^{3}\right)}^{2}+2 \operatorname{Re}\left(\eta_{s} \eta_{s}^{\prime} \phi_{m}, \frac{\partial \phi_{m}}{\partial z}\right)_{L^{2}\left(\mathbf{R}^{3}\right)}+\left\|\eta_{s} \frac{\partial \phi_{m}}{\partial z}\right\|_{L^{2}\left(\mathbf{R}^{3}\right)}^{2}
$$

The first term of the right-hand side of (4.15) is equal to $c_{0} s^{-2}$. By differentiating in $z$ the both-sides of the equality $\left\|\phi_{m}\right\|_{L^{2}\left(\mathbf{R}^{2}\right)}^{2}=1$, we have

$$
2 \operatorname{Re} \int_{\mathbf{R}^{2}} \phi_{m}(\vec{\rho}, z) \overline{\frac{\partial \phi_{m}}{\partial z}(\vec{\rho}, z)} d \vec{\rho}=0 .
$$

Hence the second term of the right-hand side of (4.15) vanishes. So we have

$$
\left\|\frac{\partial \phi_{m}^{s}}{\partial z}\right\|^{2}=c_{0} s^{-2}+\int_{\mathbf{R}^{1}}\left|\eta_{s}(z)\right|^{2}\left(\int_{\mathbf{R}^{2}}\left|\frac{\partial \phi_{m}}{\partial z}(\vec{\rho}, z)\right|^{2} d \vec{\rho}\right) d z .
$$

Now we estimate the second term of the right-hand side of (4.16). By Lemmas 4.9, 4.10 and 4.11 , and by putting $s=k^{-1} m^{1 / 8}$,

$$
\begin{aligned}
& \int_{\mathbf{R}^{2}}\left|\frac{\partial \phi_{m}}{\partial z}(\vec{\rho}, z)\right|^{2} d \vec{\rho} \leq|z|^{2}\left\{\sqrt{\frac{B}{2}} m^{-1 / 2}-\sqrt{\frac{B-2}{2}}(m+1)^{-1 / 2}+O\left(m^{-5 / 4}\right)\right\} \\
& \leq|z|^{2}\left\{\frac{1}{\sqrt{2(B-2)}}(m+1)^{-1 / 2}\left(1+\frac{B}{2 m}\right)+O\left(m^{-5 / 4}\right)\right\} \text {. }
\end{aligned}
$$

Since supp $\eta_{s} \subset\left\{z \in \mathbf{R}^{1} ; s \leq z \leq 2 s\right\}$,

[the second term of $(4.16)] \leq 4 s^{2}\left\{\frac{1}{\sqrt{2(B-2)}}(m+1)^{-1 / 2}\left(1+\frac{B}{2 m}\right)+O\left(m^{-5 / 4}\right)\right\}$ 


$$
\leq \frac{2}{k^{2}} \sqrt{\frac{2}{B-2}}(m+1)^{-1 / 4}\left(1+\frac{B}{2 m}\right)+O\left(m^{-1}\right) .
$$

Let $\varepsilon$ be an arbitrary positive number. Then for $m \geq \frac{B}{2 \varepsilon}$

(4.17) [the second term of $(4.16)] \leq(1+\varepsilon) \frac{2}{k^{2}} \sqrt{\frac{2}{B-2}}(m+1)^{-1 / 4}+O\left(m^{-1}\right)$. Hence by substituting $s=k^{-1} m^{1 / 8}$ into (4.16) and by (4.17) we obtain the first inequality of (4.10). Also recalling the definition $k=\left\{\frac{2}{c_{0}} \sqrt{\frac{2}{B-2}}\right\}^{1 / 4}$, we obtain the second equality of (4.10).

By using Lemmas 4.7 and 4.11 we now proceed to prove Proposition 4.2.

Proof of Proposition 4.2. The sequence of functions $\left\{\phi_{m}\right\}$ constructed as before satisfies $\left(\phi_{i}, \phi_{j}\right)_{L^{2}\left(\mathbf{R}^{3}\right)}=\delta_{i j}$ by Lemma 4.5 . By Lemma 4.5 again,

$$
\begin{aligned}
\left\|T \phi_{m}\right\|_{L^{2}\left(\mathbf{R}^{3}\right)}^{2} & =\left(\left(T^{2}-\left(-\partial^{2} / \partial z^{2}\right)\right) \phi_{m}, \phi_{m}\right)_{L^{2}\left(\mathbf{R}^{3}\right)}+\left\|\frac{\partial \psi_{m}}{\partial z}\right\|_{L^{2}\left(\mathbf{R}^{3}\right)}^{2} \\
& =\left(G_{f}(y) \phi_{m}, \phi_{m}\right)_{L^{2}\left(\mathbf{R}^{3}\right)}+\left\|\frac{\partial \phi_{m}}{\partial z}\right\|_{L^{2}\left(\mathbf{R}^{3}\right)}^{2}
\end{aligned}
$$

Since $k$ will be determined to be independent of $m$ later in this proof, we can assume $s(m, k) \geq 2$ if $m$ is sufficiently large. By (4.5) (if $s(m, k) \geq 2$ ),

$$
\left(G_{f}(y) \phi_{m}, \psi_{m}\right)_{L^{2}\left(\mathbf{R}^{3}\right)} \leq B+\frac{3}{2}\left(g(r) \phi_{m}, \phi_{m}\right)_{L^{2}\left(\mathbf{R}^{3}\right)},
$$

where $g(r)=f(r)-B / 2$. By Lemma 4.11,

$$
\left(g(r) \psi_{m}, \psi_{m}\right)_{L^{2}\left(\mathbf{R}^{3}\right)} \leq-\left(\frac{B-2}{2}\right)^{1 / 4}(m+1)^{-1 / 4}+O\left(m^{-1}\right),
$$

so we have

$$
\left(G_{f}(y) \phi_{m}, \phi_{m}\right)_{L^{2}\left(\mathbf{R}^{3}\right)} \leq B-\frac{3}{2}\left(\frac{B-2}{2}\right)^{1 / 4}(m+1)^{-1 / 4}+O\left(m^{-1}\right) .
$$

Using Lemma 4.7, we have for any $\varepsilon>0$

$$
\left\|T \phi_{m}\right\|_{L^{2}\left(\mathbf{R}^{3}\right)}^{2} \leq B-\left\{\frac{3}{2}\left(\frac{B-2}{2}\right)^{1 / 4}-2(1+\varepsilon) \sqrt{2 c_{0}}\left(\frac{2}{B-2}\right)^{1 / 4}\right\}(m+1)^{-1 / 4}+O\left(m^{-1}\right)
$$


when $m$ is sufficiently large. By the definition of $B$ (in (4.3)),

$$
\begin{aligned}
\frac{3}{2}\left(\frac{B-2}{2}\right)^{1 / 4}-2 \sqrt{2 c_{0}}\left(\frac{2}{B-2}\right)^{1 / 4} & =\frac{3}{2}\left(\frac{B-2}{2}\right)^{1 / 4}\left(1-\frac{8}{3} \sqrt{\frac{c_{0}}{B-2}}\right) \\
& \geq \varepsilon_{1}\left(\frac{B-2}{2}\right)^{1 / 4}>0,
\end{aligned}
$$

where $\varepsilon_{1}$ depends only on $c_{0}$ and $B$. Hence we have

$\left\|T \psi_{m}\right\|_{L^{2}\left(\mathbf{R}^{3}\right)}^{2} \leq B-\left\{\varepsilon_{1}\left(\frac{B-2}{2}\right)^{1 / 4}-2 \varepsilon \sqrt{2 c_{0}}\left(\frac{2}{B-2}\right)^{1 / 4}\right\}(m+1)^{-1 / 4}+O\left(m^{-1}\right)(m \gg 1)$.

Since $\varepsilon$ is arbitrary, we can put $\varepsilon=\frac{\varepsilon_{1}}{8} \sqrt{\frac{B-2}{c_{0}}}$. Then

$$
\begin{aligned}
\left\|T \psi_{m}\right\|_{L^{2}\left(\mathbf{R}^{3}\right)}^{2} & \leq B-\frac{\varepsilon_{1}}{2}\left(\frac{B-2}{2}\right)^{1 / 4}(m+1)^{-1 / 4}+O\left(m^{-1}\right) \\
& \leq B-\frac{\varepsilon_{1}}{4}\left(\frac{B-2}{2}\right)^{1 / 4} m^{-1 / 4}
\end{aligned}
$$

when $m$ is sufficiently large. Moreover, we can choose a subsequence of $\left\{\phi_{m}\right\}$ (denoted by the same notation) with mutually disjoint supports. Then the sequence of functions $\left\{\psi_{m}\right\}$ satisfies $\left(T \psi_{i}, T \psi,\right)_{L^{2}\left(\mathbf{R}^{3}\right)}=0$ if $i \neq j$.

To complete the proof of Proposition 4.2 we prove Lemmas 4.8-11.

Proof of Lemma 4.8. By the definition of $\beta_{m}(z)$,

$$
2 \pi \beta_{m}(z)^{2} \int_{0}^{\infty} \rho^{2 m+1} e^{-2 F(\rho, z)} d \rho=1 .
$$

By differentiating in $z$ the both-sides of (4.19),

$$
2 \pi \beta_{m}(z) \beta_{m}^{\prime}(z) \int_{0}^{\infty} \rho^{2 m+1} e^{-2 F(\rho, z)} d \rho=2 \pi \beta_{m}(z)^{2} \int_{0}^{\infty} \rho^{2 m+1} e^{-2 F(\rho, z)} \frac{\partial F}{\partial z}(\rho, z) d \rho
$$

By multiplying the both-sides of (4.20) by $\beta_{m}(z)$ and by using (4.19),

$$
\beta_{m}^{\prime}(z)=2 \pi \beta_{m}(z)^{3} \int_{0}^{\infty} \rho^{2 m+1} e^{-2 F(\rho, z)} \frac{\partial F}{\partial z}(\rho, z) d \rho .
$$

We compute $\frac{\partial F}{\partial z}$ as follows. 


$$
\begin{aligned}
\frac{\partial F}{\partial z}(\rho, z) & =\int_{0}^{\rho} f^{\prime}\left(\sqrt{t^{2}+z^{2}}\right) \frac{t z}{\sqrt{t^{2}+z^{2}}} d t \\
& =z \int_{0}^{\rho} \frac{\partial\left(f\left(\sqrt{t^{2}+z^{2}}\right)\right)}{\partial t} d t=Z(f(r)-f(|z|) .
\end{aligned}
$$

Thus we have (4.11).

Proof of Lemma 4.9. As is easily seen,

$$
\frac{\partial \phi_{m}}{\partial z}(\vec{\rho}, z)=e^{i m \theta} \rho^{m} e^{-F(\rho, z)}\left\{\beta_{m}^{\prime}(z)-\beta_{m}(z) z(f(r)-f(|z|))\right\} .
$$

Here we have used (4.21). Hence

$$
\begin{aligned}
\left|\frac{\partial \phi_{m}}{\partial z}(\vec{\rho}, z)\right|^{2}= & \rho^{2 m} e^{-2 F(\rho, z)}\left\{\beta_{m}^{\prime}(z)^{2}-2 \beta_{m}(z) \beta_{m}^{\prime}(z) z(f(r)-f(|z|))\right. \\
& \left.+\beta_{m}(z)^{2}|z|^{2}(f(r)-f(|z|))^{2}\right\}\left(\equiv\left(J_{1}\right)+\left(J_{2}\right)+\left(J_{3}\right)\right) .
\end{aligned}
$$

By use of Lemma 4.8 and (4.19),

$$
\begin{gathered}
\int_{0}^{\infty}\left(J_{1}\right) \rho d \rho=\left(2 \pi \beta_{m}(z)^{3} z\right)^{2}\left\{\int_{0}^{\infty} \rho^{2 m+1} e^{-2 F(\rho, z)}(f(r)-f(|z|)) d \rho\right\}^{2} \int_{0}^{\infty} \rho^{2 m+1} e^{-2 F(\rho, z)} d \rho \\
=2 \pi \beta_{m}(z)^{4}|z|^{2}\left\{\int_{0}^{\infty} \rho^{2 m+1} e^{-2 F(\rho, z)}(f(r)-f(|z|)) d \rho\right\}^{2}, \\
\int_{0}^{\infty}\left(J_{2}\right) \rho d \rho=-4 \pi \beta_{m}(z)^{4}|z|^{2}\left\{\int_{0}^{\infty} \rho^{2 m+1} e^{-2 F(\rho, z)}(f(r)-f(|z|)) d \rho\right\}^{2} .
\end{gathered}
$$

Now letting $g(\zeta)=f(\zeta)-B / 2(\leq 0)$ and summing up, we have

$$
\begin{aligned}
& \int_{\mathbf{R}^{2}}\left|\frac{\partial \phi_{m}}{\partial z}(\rho, z)\right|^{2} d \vec{\rho}=-\left(2 \pi \beta_{m}(z)^{2}|z|\right)^{2}\left\{\int_{0}^{\infty} \rho^{2 m+1} e^{-2 F(\rho, z)}(g(r)-g(|z|)) d \rho\right\}^{2} \\
& +2 \pi \beta_{m}(z)^{2}|z|^{2} \int_{0}^{\infty} \rho^{2 m+1} e^{-2 F(\rho, z)}(g(r)-g(|z|))^{2} d \rho \\
& =|z|^{2}\left\{\int_{0}^{\infty} 2 \pi \beta_{m}(z)^{2} \rho^{2 m+1} e^{-2 F(\rho, z)} g(r)^{2} d \rho-\left(\int_{0}^{\infty} 2 \pi \beta_{m}(z)^{2} \rho^{2 m+1} e^{-2 F(\rho, z)} g(r) d \rho\right)^{2}\right\} .
\end{aligned}
$$

Here we have used (4.19) for this calculation.

Proof of Lemma 4.10. In view that

$$
|g(r)| \leq|g(\rho)| \leq \rho^{-1 / 2}(0<\rho<+\infty)
$$

and by using (4.19) we have

[the left-hand side of (4.13)] 


$$
\begin{aligned}
& \leq 2 \pi \beta_{m}(z)^{2} \int_{0}^{\infty} \rho^{2 m} e^{-2 F(\rho, z)} d \rho \\
& \leq\left\{2 \pi \beta_{m}(z)^{2} \int_{0}^{\infty} \rho^{2 m+1} e^{-2 F(\rho, z)} d \rho\right\}^{1 / 2}\left\{2 \pi \beta_{m}(z)^{2} \int_{0}^{\infty} \rho^{2 m-1} e^{-2 F(\rho, z)} d \rho\right\}^{1 / 2} \\
& =\left\{2 \pi \beta_{m}(z)^{2} \int_{0}^{\infty} \rho^{2 m-1} e^{-2 F(\rho, z)} d \rho\right\}^{1 / 2}\left(\equiv\{(\mathrm{I})\}^{1 / 2}\right)
\end{aligned}
$$

Now we estimate (I). It follows from (4.24) that $2 F(\rho, z) \rightarrow+\infty$ as $\rho \rightarrow+\infty$. Hence by integration by parts,

$$
\begin{aligned}
(\mathrm{I}) & =2 \pi \beta_{m}(z)^{2} m^{-1} \int_{0}^{\infty} f(r) \rho^{2 m+1} e^{-2 F(\rho, z)} d \rho \\
& \leq(B / 2) m^{-1} \cdot 2 \pi \beta_{m}(z)^{2} \int_{0}^{\infty} \rho^{2 m+1} e^{-2 F(\rho, z)} d \rho \\
& =(B / 2) m^{-1} .
\end{aligned}
$$

Here we have used (4.19) and the fact that $f(r) \leq B / 2$. Summing up we get [the left-hand side of $(4.13)] \leq \sqrt{B / 2} m^{-1 / 2}$.

Proof of Lemma 4.11. We divide the left-hand side of (4.14) as follows.

[the left-hand side of (4.14)] $=2 \pi \beta_{m}(z)^{2} \int_{0}^{\infty}(-g(r)+g(\rho)) \rho^{2 m+1} e^{-2 F(\rho, z)} d \rho$

$$
+2 \pi \beta_{m}(z)^{2} \int_{0}^{\infty}(-g(\rho)) \rho^{2 m+1} e^{-2 F(\rho, z)} d \rho \equiv \text { (I) }+ \text { (II). }
$$

First we estimate (I). For $\rho \geq 2$.

$$
\begin{aligned}
0 \leq g(r)-g(\rho) & =\int_{0}^{z} \frac{\partial g\left(\sqrt{\rho^{2}+t^{2}}\right)}{\partial t} d t=\int_{0}^{z} g^{\prime}\left(\sqrt{\rho^{2}+t^{2}}\right) \frac{t}{\sqrt{\rho^{2}+t^{2}}} d t \\
& \leq \frac{z}{2} \int_{0}^{z}\left(\sqrt{\rho^{2}+t^{2}}\right)^{-5 / 2} d t \leq \frac{1}{2} z^{2} \rho^{-5 / 2}
\end{aligned}
$$

so we have

$$
-g(r)+g(\rho) \geq-\frac{1}{2} z^{2} \rho^{-5 / 2} \text { for } \rho \geq 2 \text {. }
$$

A similar method as above with (4.24) yields this inequality also for $\rho>0$. By this inequality and by Hölder's one,
(I) $\geq-\frac{z^{2}}{2} 2 \pi \beta_{m}(z)^{2} \int_{0}^{\infty} \rho^{2 m-3 / 2} e^{-2 F(\rho, z)} d \rho$ 


$$
\geq-\frac{z^{2}}{2} 2 \pi \beta_{m}(z)^{2}\left(\int_{0}^{\infty} \rho^{2 m-3} e^{-2 F(\rho, z)} d \rho\right)^{1 / 4} \cdot\left(\int_{0}^{\infty} \rho^{2 m-1} e^{-2 F(\rho, z)} d \rho\right)^{3 / 4} .
$$

By integration by parts and by the fact that $f(r) \leq B / 2$ we have

$$
\begin{aligned}
\int_{0}^{\infty} \rho^{2 m-3} e^{-2 F(\rho, z)} d \rho & =(m-1)^{-1} \int_{0}^{\infty} \rho^{2 m-1} f(r) e^{-2 F(\rho, z)} d \rho \\
& \leq(B / 2)(m-1)^{-1} \int_{0}^{\infty} \rho^{2 m-1} e^{-2 F(\rho, z)} d \rho .
\end{aligned}
$$

Hence by use of the estimation of (I) in the proof of Lemma 4.10 we have

$$
\text { (I) } \begin{aligned}
\geq & -(B / 2)^{1 / 4}(m-1)^{-1 / 4}\left(z^{2} / 2\right) 2 \pi \beta_{m}(z)^{2} \int_{0}^{\infty} \rho^{2 m-1} e^{-2 F(\rho, z)} d \rho \\
& \geq-(B / 4) m^{-1}(B / 2)^{1 / 4}(m-1)^{-1 / 4} z^{2} \\
& \geq-B s^{2} m^{-1}(B / 2)^{1 / 4}(m-1)^{-1 / 4}
\end{aligned}
$$

for $s \leq z \leq 2 s$. By putting $s=k^{-1} m^{1 / 8}$,

$$
\text { (I) } \geq-B k^{-2} m^{-1}(B / 2)^{1 / 4}\left(\frac{m}{m-1}\right)^{1 / 4} \geq-C_{1}(B, k) m^{-1}
$$

for some positive constant $C_{1}(B, k)$ depending on $B$ and $k$.

Next we estimate (II). By the definition $g(\rho)=f(\rho)-B / 2$ and (4.4),

$$
\begin{aligned}
(\mathrm{II}) & =2 \pi \beta_{m}(z)^{2}\left\{\int_{0}^{\infty} \rho^{2 m+1 / 2} e^{-2 F(\rho, z)} d \rho+\int_{0}^{2}\left(-g(\rho) \rho^{2 m+1}-\rho^{2 m+1 / 2}\right) e^{-2 F(\rho, z)} d \rho\right\} \\
& \geq 2 \pi \beta_{m}(z)^{2}\left\{\int_{0}^{\infty} \rho^{2 m+1 / 2} e^{-2 F(\rho, z)} d \rho-C_{2} \int_{0}^{2} \rho^{2 m} e^{-2 F(\rho, z)} d \rho\right\},
\end{aligned}
$$

where $C_{2}=\sup _{0 \leq \rho \leq 2}\left(|g(\rho)| \rho+\rho^{1 / 2}\right)$. By use of Lemma 4.6 with $R=4$ and the estimate $\int_{0}^{2} \rho^{2 m} e^{-2 F(\rho, z)} d \rho \leq 2^{2 m+1}$, we have

$$
\begin{aligned}
\text { (II) } & \geq 2 \pi \beta_{m}(z)^{2} \int_{0}^{\infty} \rho^{2 m+1 / 2} e^{-2 F(\rho, z)} d \rho-2 \pi C_{2} 2^{-2 m-1} \\
& \geq 2 \pi \beta_{m}(z)^{2} \int_{0}^{\infty} \rho^{2 m+1 / 2} e^{-2 F(\rho, z)} d \rho-C_{3}(B) m^{-1}
\end{aligned}
$$

for some positive constant $C_{3}(B)$. We now estimate the first term of the right-hand side of (4.26). By use of (4.19) and Schwarz inequality,

$$
\begin{aligned}
1 & =\left(2 \pi \beta_{m}(z)^{2}\right)^{2}\left(\int_{0}^{\infty} \rho^{2 m+1} e^{-2 F(\rho, z)} d \rho\right)^{2} \\
& \leq\left(2 \pi \beta_{m}(z)^{2}\right)^{2} \int_{0}^{\infty} \rho^{2 m+1 / 2} e^{-2 F(\rho, z)} d \rho \int_{0}^{\infty} \rho^{2 m+3 / 2} e^{-2 F(\rho, z)} d \rho .
\end{aligned}
$$


Furthermore by using Hölder's inequality and by using (4.19) again, we have

$$
\begin{aligned}
& \left(2 \pi \beta_{m}(z)^{2} \int_{0}^{\infty} \rho^{2 m+1 / 2} e^{-2 F(\rho, z)} d \rho\right)^{-1} \\
\leq & 2 \pi \beta_{m}(z)^{2} \int_{0}^{\infty} \rho^{2 m+3 / 2} e^{-2 F(\rho, z)} d \rho \\
\leq & 2 \pi \beta_{m}(z)^{2}\left(\int_{0}^{\infty} \rho^{2 m+3} e^{-2 F(\rho, z)} d \rho\right)^{1 / 4}\left(\int_{0}^{\infty} \rho^{2 m+1} e^{-2 F(\rho, z)} d \rho\right)^{3 / 4} \\
= & \left(2 \pi \beta_{m}(z)^{2} \int_{0}^{\infty} \rho^{2 m+3} e^{-2 F(\rho, z)} d \rho\right)^{1 / 4} .
\end{aligned}
$$

Using the fact that $B / 2-1 \leq f(r) \leq B / 2(0 \leq r<+\infty)$ and integrating by parts, we have

$$
\begin{aligned}
\int_{0}^{\infty} \rho^{2 m+3} e^{-2 F(\rho, z)} d \rho & \leq \int_{0}^{\infty}(B / 2-1)^{-1} f(r) \rho^{2 m+3} e^{-2 F(\rho, z)} d \rho \\
& =-(B-2)^{-1} \int_{0}^{\infty} \rho^{2 m+2} \frac{\partial\left(e^{-2 F(\rho, z)}\right)}{\partial \rho} d \rho \\
& =2(m+1)(B-2)^{-1} \int_{0}^{\infty} \rho^{2 m+1} e^{-2 F(\rho, z)} d \rho .
\end{aligned}
$$

Hence we have by (4.27), (4.28) and (4.19)

$$
2 \pi \beta_{m}(z)^{2} \int_{0}^{\infty} \rho^{2 m+1 / 2} e^{-2 F(\rho, z)} d \rho \geq\left(\frac{B-2}{2}\right)^{1 / 2}(m+1)^{-1 / 4} .
$$

Combining (4.25) with (4.26), (4.29) we obtain (4.14).

\section{Proof of Theorem 1.1}

In this section, using propositions and lemmas in previous sections we prove Theorem 1.1. At first we show the following proposition.

PROPOSITION 5.1. We recall the vector potential $b_{0}$ defined in $\S 4$ and suppose that

$$
\sigma_{d}\left(H_{N-1, Z}\left(b_{0}\right)\right) \neq \emptyset .
$$

Then there exists a sequence of functions $\left\{\Phi_{m}\right\} \subset Q\left(H_{N, Z}\left(b_{0}\right)\right)$ such that

$$
\left\{\begin{array}{l}
\left(\Phi_{i}, \Phi_{j}\right)_{L^{2}\left(\mathbf{R}^{3}\right)}=\delta_{i j}, \\
q_{H_{N}}\left[\Phi_{i}, \Phi_{j}\right]=0 \text { if } i \neq j \text { and } \\
q_{H_{N}}\left[\Phi_{m}\right]<B+\Lambda\left(H_{N-1}\right),
\end{array}\right.
$$


where $H_{N}=H_{N, Z}\left(b_{0}\right)$ and $Q\left(H_{N}\right)$ denotes the form domain of $H_{N}$.

Proof of Proposition 5.1. Let $x=\left(x^{\prime}, x^{N}\right) \in \mathbf{R}^{3 N}, x^{\prime}=\left(x^{1}, \ldots, x^{N-1}\right) \in$ $\mathbf{R}^{3(N-1)}$ and pick up a normalized eigenfunction $\eta\left(x^{\prime}\right)$ corresponding to the ground state energy $\Lambda\left(H_{N-1}\right)$. We note that in view of (5.1) the ground state energy belongs to the discrete spectrum of $H_{N-1}$. Using the sequence of functions $\left\{\phi_{m}\right\}_{m}$ in Proposition 4.2 , we define the sequence of functions $\left\{\Phi_{m}\right\}_{m} \subset Q\left(H_{N}\right)$ by

$$
\Phi_{m}(x)=\eta\left(x^{\prime}\right) \phi_{m}\left(x^{N}\right) \quad(m \in \mathbf{N}) .
$$

These functions satisfy

$$
\left(\Phi_{i}, \Phi_{j}\right)_{L^{2}\left(\mathbf{R}^{3 N)}\right.}=\delta_{i j}(i, j \in \mathbf{N})
$$

because of the orthonormal property of $\left\{\psi_{m}\right\}_{m}$. Now using the equality:

$$
H \equiv H_{N, Z}\left(b_{0}\right)=H_{N-1, Z}\left(b_{0}\right)+T_{N}^{2}-\frac{Z}{\left|x^{N}\right|}+\sum_{j=1}^{N-1} \frac{1}{\left|x^{j}-x^{N}\right|},
$$

we have by dropping the third term of (5.4)

$$
\begin{aligned}
q_{H_{N}}\left[\Phi_{m}\right] & \leq q_{H_{N-1}}[\eta]+\left\|T_{N} \psi_{m}\right\|_{L^{2}\left(\mathbf{R}^{3}\right)}^{2}+\sum_{j=1}^{N-1}\left(\frac{1}{\left|x^{j}-x^{N}\right|} \Phi_{m}, \Phi_{m}\right)_{L^{2}\left(\mathbf{R}^{3 N}\right)} \\
& \leq \Lambda\left(H_{N-1}\right)+B-\varepsilon_{0} m^{-1 / 4}+\sum_{j=1}^{N-1}\left(\frac{1}{\left|x^{j}-x^{N}\right|} \Phi_{m}, \Phi_{m}\right)_{L^{2}\left(\mathbf{R}^{3 N}\right)}
\end{aligned}
$$

when $m$ is sufficiently large. Here we have used Proposition 4.2.

In order to estimate the last term of the right-hand side of (5.5), we divide it into two parts as follows.

$$
\begin{aligned}
\left(\frac{1}{\left|x^{j}-x^{N}\right|} \Phi_{m}, \Phi_{m}\right)_{L^{2}\left(\mathbf{R}^{3 N}\right)} & =\left\{\int_{\left|x^{j}\right| \leq\left|x^{N}\right| / 2}+\int_{\left|x^{j}\right|>\left|x^{N}\right| / 2}\right\} \frac{1}{\left|x^{j}-x^{N}\right|}\left|\Phi_{m}\right|^{2} d x \\
& \equiv(\mathrm{I})+(\mathrm{II}) .
\end{aligned}
$$

First we consider (I). Since $\left|x^{i}-x^{j}\right| \geq\left|x^{N}\right| / 2$ on the integral region of (I),

$$
\text { (I) } \leq 2 \int_{\left|x^{j}\right| \leq\left|x^{N}\right| / 2} \frac{1}{\left|x^{N}\right|}\left|\Phi_{m}(x)\right|^{2} d x \leq 2\left(\left|x^{N}\right|^{-1} \Phi_{m}, \Phi_{m}\right)_{L^{2}\left(\mathbf{R}^{3 N)}\right.} \leq 2\left(\rho_{N}^{-1} \psi_{m}, \phi_{m}\right)_{L^{2}\left(\mathbf{R}^{3}\right)},
$$

where $x^{N}=\left(x_{1}^{N}, x_{2}^{N}, x_{3}^{N}\right)$ and $\rho_{N}=\left\{\left(x_{1}^{N}\right)^{2}+\left(x_{2}^{N}\right)^{2}\right\}^{1 / 2}$. By the estimation in the proof of Lemma 4.10 we have

$$
\text { (I) } \leq 2 \sqrt{2 B} m^{-1 / 2} \text {. }
$$


Next we consider (II). Since $\left|x^{j}\right|>\left|x^{N}\right| / 2$,

$$
\begin{aligned}
& (\mathrm{II}) \leq \int_{\mathbf{R}^{3}}\left\{\int_{\mathbf{R}^{3(N-1)}} \frac{1}{\left|x^{j}-x^{N}\right|} \eta\left(x^{\prime}\right) \overline{\eta\left(x^{\prime}\right)} e^{\delta_{0}\left|x^{j}\right|} d x^{\prime}\right\} \bar{e}^{\delta_{0}\left|x^{N}\right| / 2}\left|\phi_{m}\left(x^{N}\right)\right|^{2} d x^{N} \\
\leq & \int_{\mathbf{R}^{3}}\left\|\frac{1}{\left|x^{j}-x^{N}\right|} \eta\left(x^{\prime}\right)\right\|_{L^{2}\left(\mathbf{R}^{3(N-1)}\right)} \cdot\left\|\eta\left(x^{\prime}\right) e^{\delta_{0}\left|x^{j}\right|}\right\|_{L^{2}\left(\mathbf{R}^{3(N-1)}\right)} \cdot \bar{e}^{\delta_{0}\left|x^{N}\right| / 2}\left|\phi_{m}\left(x^{N}\right)\right|^{2} d x^{N} .
\end{aligned}
$$

Here $\delta_{0}$ is in Theorem 3.1 , which states $L^{2}$-exponential decay of eigenfunctions, so

$$
\left\|\eta\left(x^{\prime}\right) e^{\delta_{0}\left|x^{j}\right|}\right\|_{L^{2}\left(\mathbf{R}^{3(N-1)}\right)} \leq C_{1}<+\infty
$$

for some positive constant $C_{1}$. By the uncertainty principle lemma (see [10] Lemma $4.7,[14]$ p.169) we have

$$
\left\|\frac{1}{\left|x^{j}-x^{N}\right|} \eta\left(x^{\prime}\right)\right\|_{L^{2}\left(\mathbf{R}^{3(N-1)}\right)} \leq 2\left\|T_{j} \eta\right\|_{L^{2}\left(\mathbf{R}^{3(N-1)}\right)} \equiv C_{2}<+\infty .
$$

Summing up we have

$$
\text { (II) } \leq C_{1} C_{2} \int_{\mathbf{R}^{3}} e^{-\delta_{0}\left|x^{N}\right| / 2}\left|\phi_{m}\left(x^{N}\right)\right|^{2} d x^{N}
$$

In view that $\operatorname{supp} \psi_{m} \subset\left\{x^{N} \in \mathbf{R}^{3} ;\left|x^{N}\right| \geq k^{-1} m^{1 / 8}\right\}$ ( $k$ is defined the proof of Proposition 2),

$$
\text { (II) } \leq C_{1} C_{2} e^{-\delta_{0} k^{-1} m^{1 / 8} / 2} \int_{\mathbf{R}^{3}}\left|\psi_{m}\right|^{2} d x^{N}=O\left(e^{-\delta_{0} k^{-1} m^{1 / 8} / 2}\right) .
$$

Combining (5.6) with (5.7) yields

$$
\left(\frac{1}{\left|x^{j}-x^{N}\right|} \Phi_{m}, \Phi_{m}\right)_{L^{2}\left(\mathbf{R}^{3 N)}\right.}=O\left(m^{-1 / 2}\right)
$$

hence by going back to (5.5) we obtain

$$
q_{H_{N}}\left[\Phi_{m}\right] \leq \Lambda\left(H_{N-1}\right)+B-\frac{\varepsilon_{0}}{2} m^{-1 / 4}<\Lambda\left(H_{N-1}\right)+B
$$

when $m$ is sufficient large.

In addition, by the same reason as in the proof of Proposition 4.2, there is a subsequence of $\left\{\Phi_{m}\right\}_{m}$ (denoted by the same notation) satisfying $q_{H_{N}}\left[\Phi_{i}, \Phi_{j}\right]=0$ if $i \neq j$. Hence $\left\{\Phi_{m}\right\}_{m}$ satisfies (5.2).

We now prove the main theorem by using Rayleigh-Ritz method. 
Proof of Theorem 1.1. Let $b=b_{0}$ in $\S 4$. This $b_{0}$ is independent of $N$ and $Z$. We show that the vector potential $b_{0}$ satisfies the statement of Theorem 1.1 , by using an induction with respect to $N$ when $Z$ is fixed. For the sake of convenience we denote $H_{N, Z}\left(b_{0}\right)$ by $H_{N}$.

First we consider the case $N=1$. From Theorems 2.1 and 2.6 it follows that $\Sigma\left(H_{1}\right)=B$. Here we have used the fact that $-\frac{Z}{|y|}$ decays at infinity (see also [9] §2). By Theorem 4.1 and by the fact that $T_{1}^{2} \leq H_{1}$, we have $\# \sigma_{d}\left(H_{1}\right)=+\infty$.

Next suppose that

$$
\sum\left(H_{l}\right)=\Lambda\left(H_{l-1}\right)+B(1 \leq l \leq N-1) \text { and } \# \sigma_{d}\left(H_{N-1}\right)=+\infty .
$$

Then by Theorem 2.8 we have

$$
\sum\left(H_{N}\right) \geq \Lambda\left(H_{N-1}\right)+B .
$$

By (5.8), Proposition 5.1 and Rayleigh-Ritz method, we have

$$
\#\left\{\sigma_{d}\left(H_{N}\right) \cap\left(-\infty, \Sigma\left(H_{N}\right)\right)\right\}=+\infty .
$$

Also it follows that $\sum\left(H_{N}\right)=B+\Lambda\left(H_{N-1}\right)$. We remark that $\sigma_{e}\left(H_{N}\right)=\left[\sum\left(H_{N}\right)\right.$, $\infty)$ by the same method as in [10]. This completes the proof of Theorem 1.1.

Acknowledgement. It is great pleasure to thank Professor H. Isozaki for giving me many advices through a series of my works.

\section{REFERENCES}

[1] S. Agmon, Bounds on exponential decay of eigenfunctions of Schrödinger operators, in Schrödinger operators, ed. by S. Graffi, Lecture Note in Math., 1159, Springer (1985).

[2] S. Agmon, Lectures on exponential decay of solutions of second-order elliptic equations: Bounds on eigenfunctions of $N$-body Schrödinger operators, Math. Notes, 29, Princeton University Press and the University of Tokyo Press (1982).

[ 3 ] J. Avron, I. Herbst, B. Simon, Schrödinger operators with magnetic fields I, General Interactions, Duke Math. J., 45 (1978), 847-883.

[4] J. Avron, I. Herbst, B. Simon, Schrödinger operators with magnetic fields III, Atoms in homogeneous magnetic field, Comm. Math. Phys., 79 (1981), 529-572.

[5] R. Carmona, B. Simon, Pointwise bound on eigenfunctions and wave packets in $N$-body quantum systems, V: Lower bound and path integrals, Comm. Math. Phys., 80 (1981), 59-98.

[6] J. M. Combes, L. Thomas, Asymptotic behavior of eigenfunctions for multiparticle Schrödinger operators, Comm. Math. Phys., 34 (1973), 251-276.

[ 7 ] H. L. Cycon, R. G. Froese, W. Kirsch, B. Simon, Schrödinger operators with ap- 
plication to quantum mechanics and global geometry, Texts and Monographs in Physics, Springer-Verlag, New York/Berlin (1987).

[8] W. D. Evans, R. T. Lewis, Y. Saitō, Some geometric spectral properties of $N$-body Schrödinger operators, Arch. Ratio. Mech.Anal., 113 (1991), 377-400.

[9] T. Hattori, Discrete spectrum of Schrödinger operators with perturbed Uniform magnetic fields, Osaka J. Math, 32 (1995), 783-797.

[10] T. Hattori, Discrete spectrum of many body Schrödinger operators with non-constant magnetic fields I, preprint.

[11] E. H. Lieb, Bounds on the maximum negative ionization of atoms and molecules, Physical Review, A 29 (1984), 3018-3028.

[12] T. O'Conner, Exponential decay of bound state wave functions, Comm. Math. Phys., 32 (1973), 319-340.

[13] A. Persson, Bounds for the discrete part of the spectrum of a semi-bounded Schrödinger operator, Math. Scand, 8 (1960), 143-153.

[14] M. Reed, B. Simon, Methods of Modern Mathematical Physics II Fourier Analysis, Self-adjointness, Academic Press (1975).

[15] M. Reed, B. Simon, Methods of Modern Mathematical Physics IV Analysis of Operators, Academic Press (1978).

[16] M. B. Ruskai, Absence of discrete spectrum in highly negative ions, Comm. in Math. Phys., 82 (1982), 457-469.

[17] M. B. Ruskai, Absence of discrete spectrum in highly negative ions, II Comm. in Math. Phys., 85 (1982), 325-327.

[18] I. M. Sigal, Geometric Methods in the Quantum Many-Body Problem, Nonexistence of Very Negative Ions, Comm. in Math. Phys., 85 (1982), 309-324.

[19] B. Simon, Pointwise bounds on eigenfunctions and wave packets in $N$-body quantum systems I, Proc. Amec. Math. Soc., 42 (1974), 359-401.

[20] S. A. Vugal'ter, G. M. Zhislin, Discrete spectra of Hamiltonians of multi-particle systems in a uniform magnetic field, Sov. Phys. Dokl., 36 (1991), 299-300.

[21] D. R. Yafaev, The point spectrum in the quantum-mechanical problem of many particles, Func. Anal. Appl., 6 (1972), 349-350.

[22] D. R. Yafaev, On the point spectrum in the quantum-mechanical many -body problem, Math. USSR Isv., 10 (1976), 861-896.

[23] G. M. Zhislin, An investigation of the spectrum of differential operators of many-particle quantum-mechanical systems in function spaces of given symmetry, Math. USSR Isv., 3 (1969), 559-616.

[24] G. M. Zhislin, On the finiteness of the discrete spectrum of the energy operator of negative ions, Theor. Math. Phys., 7 (1971), 571-578.

[25] G. M. Zhislin, On the finiteness of the discrete spectrum of energy operators of multiparticle quantum systems, Sov. Math. Dokl., 13 (1972), 1445-1449.

[26] G. M. Zhislin, Finiteness of the discrete spectrum in the quantum $N$-particle problem, Theor. Math. Phys., 21 (1974), 971-990.

Department of Mathematics

Osaka University

Toyonaka, Osaka 560

Japan
Current Address:

Department of Mechanics and Applied Mathematics

Osaka Institute of Technology

5-16-1, Omiya, Asahi-ku, Osaka 535

Japan 